CERN-PPE/97-38

April 10,1997

\title{
Experimental Results on Neutrino Masses and Lepton Mixing
}

\author{
JÜRGEN BRUNNER \\ CERN
}

1211 Geneva 23, Switzerland

\begin{abstract}
The experimental status of measurements of neutrino masses and lepton flavour mixing is summarised. Limits of mass and mixing parameters obtained by different experimental techniques are given and the possibilities and limits of the different methods are discussed. Future projects are presented and their ability to verify existing results or to explore further regions of the mass and mixing parameter space is discussed.
\end{abstract}

Accepted for publication by Progress of Physics 


\section{Introduction}

After forty years of experiments with neutrinos the field has achieved a remarkable number of successful results. The discovery of neutral-current interactions, the first precise measurement of electroweak parameters such as $\sin ^{2} \theta_{W}$ and detailed measurements of nucleon structure are only some.

However, only a few of the intrinsic properties of the neutrinos themselves are known:

- Weak interactions violate parity maximally. Therefore only left-handed neutrinos and right-handed antineutrinos are "visible" via their interactions, where "handedness" denotes the chirality states. For massless neutrinos these states are identical to the corresponding helicity states, whereas for massive neutrinos the chirality states are a combination of the helicity states.

- Neutrinos carry a "lepton flavour" quantum number. This means they "remember" (at least over some finite distance) the flavour of the charged lepton which was involved in their creation process.

- There are three light neutrinos with standard couplings. This result has been obtained from the analysis of the $\mathrm{Z}$ decay [1]. Therefore two choices remain for the introduction of new neutrino flavours:

- heavy neutrinos with masses above $45 \mathrm{GeV}$, so they cannot contribute to the $\mathrm{Z}$ decays.

- sterile light neutrinos which couple much weaker to the $\mathrm{Z}$ and $\mathrm{W}$ than the standard neutrinos do. These hypothetic particles are sometimes used in the interpretation of results of neutrino oscillation experiments.

On the contrary some very important questions are not yet answered:

- Do neutrinos have a mass ?

- Is lepton flavour strictly conserved?

- Are neutrinos their own antiparticles?

- Are there more neutrino species?

The interest in these questions is driven by several inputs :

Theory Almost all extensions of the Standard Model assume massive neutrinos and mixing among the different lepton flavours. Even without going to specific new models it is the simplest and most straightforward extension of the Standard Model to include Dirac mass terms for neutrinos and a mixing scheme analogous to the quark sector.

Experiments Within the last years various experiments have seen deviations from expected Standard Model results which allow an interpretation in the frame of massive neutrinos and flavour mixing. This has created a large impetus in the field and many proposals for confirmation experiments have appeared.

Cosmology The dark matter problem is not yet solved and remains the key point in the understanding of the further evolution of the universe. Models with a mixture of hot and cold dark matter [2] seem to be most successful in describing the observed level 
of fluctuations in the cosmic microwave background radiation [3, 4]. Neutrinos with masses in the range 1-10 eV are the most probable candidates for hot dark matter.

This paper will concentrate on the experiments and will give an overview over existing limits for neutrino masses and lepton flavour mixing, some positive hints and experimental plans for the near future.

\section{Direct neutrino mass measurements}

A model-independent way to measure neutrino masses is to study decay processes with neutrinos involved. In the case of non-zero neutrino masses, the decay kinematics change, which could lead to observable effects.

\section{$2.1 \quad \bar{\nu}_{e}$ mass}

The mass of the electron neutrino (more precisely the electron-antineutrino) has been investigated using the $\beta$-decay of tritium:

$$
{ }_{1}^{3} \mathrm{H} \rightarrow{ }_{2}^{3} \mathrm{He}+e^{-}+\bar{\nu}_{e} .
$$

The advantages of this transition compared to other $\beta$-decays is the small transition energy, only $18 \mathrm{keV}$, and the absence of atomic screening effects by additional electrons. Limits for the neutrino mass are derived by a fit to the highest energy part of the electron spectrum, typically a few $100 \mathrm{eV}$ below its endpoint.

There are two experiments currently taking data which reach the highest sensitivity to detect the neutrino mass. One is carried out at the University of Mainz, the second at Troitsk in Russia. In the past both experiments reported a dependence of the fitted value of $m_{\nu}^{2}$ on the specific energy range chosen for the fit $[5,6]$. This is a clear indication of a systematic error in the description of the data. In a reanalysis of the data the Troitsk group found an underestimation of an effect which is connected with the trapping of electrons in their tritium source [7]. Applying an additional correction for this effect the fitted values of $m_{\nu}^{2}$ become independent of the energy range.

The Mainz group is still investigating similar possibilities in their setup. Most recent results are therefore restricted to a fit of the last $140 \mathrm{eV}$ below the endpoint. In this region the effect is supposed to be negligible.

Finally the Troitsk group observes a bump-like structure near the end point which changes position if one compares the data taken in 1994 and 1996. This is again an indication for an unknown systematic effect. Position and integral intensity of the bumplike structure were introduced as free parameters into the fit to obtain undistorted values for $m_{\nu}^{2}$.

The most recent published values for $m_{\nu}^{2}$ are $[7,8]$

$$
\begin{array}{ll}
\text { Mainz } & m_{\bar{\nu}_{e}}^{2}=-22 \pm 17 \pm 14 \mathrm{eV}^{2} \\
\text { Troitsk } & m_{\bar{\nu}_{e}}^{2}=1.5 \pm 5.9 \pm 3.6 \mathrm{eV}^{2} .
\end{array}
$$

which can be transformed into upper limits for the $\bar{\nu}_{e}$ - neutrino mass. For $95 \%$ confidence level one obtains :

$$
\begin{array}{ll}
\text { Mainz } & m_{\bar{\nu}_{e}}<5.6 \mathrm{eV} \\
\text { Troitsk } & m_{\bar{\nu}_{e}}<3.9 \mathrm{eV}
\end{array}
$$

However, the Particle Data Group decided not to include these numbers in its 1996 Review of Particle Properties [9] because of the above-mentioned unresolved systematic effects. 


\section{$2.2 \quad \nu_{\mu}$ mass}

The best limits for the mass of the $\nu_{\mu}$ come from the study of the decay

$$
\pi^{+} \rightarrow \mu^{+}+\nu_{\mu}
$$

with the pion at rest. This reaction can only be studied with $\pi^{+}$because $\pi^{-}$are captured by nuclei when they come to rest. This is a two-body decay and only two values influence the sensitivity with respect to the neutrino mass : the muon momentum and the pion mass (the muon mass is known with a 10 times higher precision than the pion mass).

Due to a reanalysis of x-ray spectra of pionic atoms the value of the pion mass moved by 6 sigma from the value measured in [10] to the one given in [11]:

$$
\begin{array}{ll}
\text { old value } & 139.56782 \pm 0.00037 \mathrm{MeV} \\
\text { new value } & 139.56995 \pm 0.00035 \mathrm{MeV}
\end{array}
$$

A reevaluation of a neutrino mass limit, obtained at PSI [12] in the light of the changed pion mass value gave

$$
m_{\nu}^{2}=-0.016 \pm 0.023 \mathrm{MeV}^{2}
$$

which can be transformed into an upper limit for the muon neutrino mass

$$
m_{\nu_{\mu}}<170 \mathrm{keV} ; 90 \% \text { C.L. }
$$

\section{$2.3 \quad \nu_{\tau}$ mass}

Limits for the $\nu_{\tau}$ mass have been derived from the study of the decays

$$
\begin{aligned}
& \tau \rightarrow 5 \pi\left(\pi^{o}\right)+\nu_{\tau} \\
& \tau \rightarrow 3 \pi\left(\pi^{o}\right)+\nu_{\tau} .
\end{aligned}
$$

These decay channels have been chosen to reduce the available phase space for the neutrino as much as possible. The pioneering experiment was ARGUS [13]. The neutrino mass limit was extracted from the analysis of the invariant mass of the hadronic system:

$$
\text { ARGUS } m_{\nu_{\tau}}<31 \mathrm{MeV} \text { 95\% C.L. }
$$

Two LEP experiments $[14,15]$ could improve this value by analysing the data in the two dimensional parameter space of hadronic mass and hadronic energy. The final results are:

$$
\begin{array}{lll}
\text { ALEPH } & m_{\nu_{\tau}}<23.1 \mathrm{MeV} & 95 \% \text { C.L. } \\
\text { OPAL } & m_{\nu_{\tau}}<29.9 \mathrm{MeV} & 95 \% \text { C.L. }
\end{array}
$$

\section{Double beta decay}

Observation of double beta decay is possible when single beta decay is energetically forbidden but double beta decay is allowed as indicated in figure 1 .

There are a number of isotopes which fulfill this condition. Some of the most commonly used are:

$$
{ }^{76} \mathrm{Ge},{ }^{82} \mathrm{Se},{ }^{100} \mathrm{Mo},{ }^{128} \mathrm{Te},{ }^{130} \mathrm{Te},{ }^{136} \mathrm{Xe} .
$$




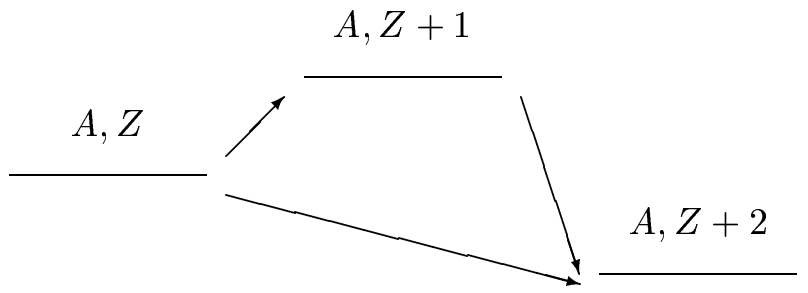

Figure 1: Energy levels of isobaric nuclei to allow observation of double beta decay

The double beta decay of the type :

$$
{ }_{Z}^{A} X \rightarrow{ }_{Z+2}^{A} Y+2 e^{-}+2 \bar{\nu}_{e}
$$

is allowed in the frame of the Standard Model as a second order process and lifetimes of the order of $10^{20}$ years are expected. The first obversation of this process in a real time counter experiment has been done for the decay

$$
{ }_{34}^{82} S e \rightarrow{ }_{36}^{82} K r+2 e^{-}+2 \bar{\nu}_{e}
$$

and a halflife of

$$
T_{1 / 2}=1.1 \cdot 10^{20} y
$$

has been measured [16] in good agreement to earlier geochemical measurements [17].

For the investigation of intrinsic neutrino properties the neutrinoless double beta decay, such as indicated in figure 2 , is much more interesting.

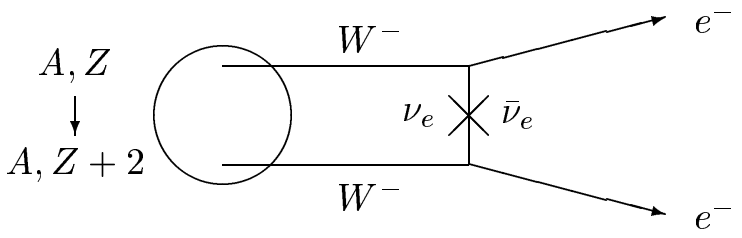

Figure 2: Schematic diagram for neutrinoless double beta decay

The essential difference with respect to the two-neutrino double beta decay is the neutrino propagator. At the emission vertex a right-handed antineutrino is needed whereas at the absorption vertex a left-handed neutrino is needed. Therefore one can derive two necessary conditions:

- $\nu=\bar{\nu}$, which implies Majorana type neutrinos

- $m_{\nu}>0$, to allow a wrong-helicity admixture in the weak coupling

The experimental signature is clear and well separated from the Standard Model double beta decay. One expects a sharp line in the spectrum of the sum of the electron energies because no energy is lost due to escaping neutrinos. 
The best limits so far have been determined by the Heidelberg-Moscow experiment [18] studying the decay

$$
{ }_{32}^{76} G e \rightarrow{ }_{34}^{76} S e+2 e^{-}+2 \bar{\nu}_{e}
$$

The experiment is carried out at the Gran Sasso laboratory in Italy. They are working with the heaviest probe (11.5 $\mathrm{kg}$ of Germanium with enriched ${ }^{76} \mathrm{Ge}$ content) compared to other experiments and latest results are based on $17.17 \mathrm{~kg} \cdot \mathrm{y}$. With an energy resolution of $1 \mathrm{keV}$ and a background of $0.21 \pm 0.01 \mathrm{cts} \cdot \mathrm{kg}^{-1} \cdot y^{-1} \cdot \mathrm{keV}^{-1}$ a limit of

$$
T_{1 / 2}>9.6 \cdot 10^{24} \text { y } \quad 90 \% \text { C.L. }
$$

is derived. Since one needs the wrong-helicity admixture of the neutrino at one of the weak decay vertices, the lifetime limit can be transformed into an upper limit for the electron neutrino mass of

$$
m_{\nu}<0.50 \mathrm{eV} \quad 90 \% \text { C.L. }
$$

This limit is one order of magnitude better than the limits which can be obtained by studying the Tritium beta decay but it is only valid under the assumption that neutrinos are Majorana particles. The interpretation of this result in the framework of several mass eigenstates is more difficult because of possible cancellation effects between the different terms.

It is planned to continue the above experiment for at least another three years. The anticipated result is expected to base on $50 \mathrm{~kg} \cdot y$ with a corresponding improvement of the sensitivity towards smaller neutrino masses.

\section{Neutrino oscillation searches}

The concept of neutrino oscillations has been introduced by Pontecorvo [19], originally for the process $\nu_{e} \leftrightarrow \bar{\nu}_{e}$. After the discovery of $\nu_{\mu}$ this concept has been adapted to transitions between different neutrino flavours [20] as they are discussed in the following sections.

\subsection{Vacuum oscillations}

To observe neutrino oscillations two conditions have to be fulfilled :

- violation of lepton flavour number

- massive neutrinos

The most straightforward way to introduce these conditions is to assume that the flavour eigenstates $\left(\nu_{\alpha}\right)$ which take part into the weak interactions are linear combinations of mass eigenstates $\left(\nu_{i}\right)$ which describe the propagation of the neutrino field in space time

$$
\nu_{i}=\sum_{i} U_{\alpha i} \nu_{\alpha}
$$

Here $U_{\alpha i}$ denotes the unitary mixing matrix. This concept has been successfully applied in the quark sector, where $U_{\alpha i}$ is known as Cabibbo-Kobayashi-Maskawa matrix.

In an oscillation experiment one creates a neutrino beam of defined weak eigenstate $\nu_{\alpha}$ at $t=x=0$ and tries to detect at a different point of space time the weak eigenstate 


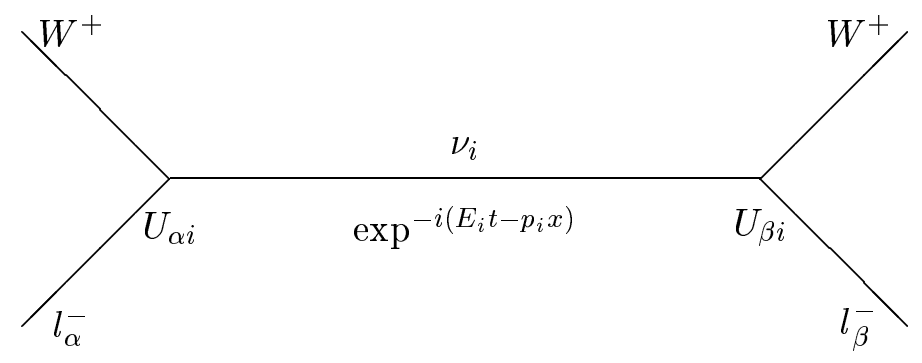

Figure 3: Schematic diagram of an oscillation experiment

$\nu_{\beta}$ (not necessarily $\alpha \neq \beta$ ) as shown in figure 3 . The transition probability can be written as

$$
P(\alpha \rightarrow \beta)=\left|\sum_{i} U_{\beta i} \exp ^{-i\left(E_{i} t-p_{i} x\right)} U_{\alpha i}^{*}\right|^{2} .
$$

The rightmost factor describes the probability to materialise as mass eigenstate $i$ at the neutrino source, the exponential factor describes the propagation of the mass eigenstate in space-time (in vacuum) and the leftmost factor gives the probability to interact as weak eigenstate $\beta$ at the neutrino detector. A simple transformation leads to

$$
P(\alpha \rightarrow \beta)=\sum_{i}\left|U_{\beta i}\right|^{2}\left|U_{\alpha i}\right|^{2}+R e \sum_{i \neq j} U_{\beta i} U_{\beta j}^{*} U_{\alpha i}^{*} U_{\alpha j} \exp ^{-i\left[\left(E_{i}-E_{j}\right) t-\left(p_{i}-p_{j}\right) x\right]}
$$

The first term describes the classical transition probability from weak eigenstate $\alpha$ into $\beta$ via all possible mass eigenstates $i$ whereas the second term contains the quantummechanical phase information leading to an $(x, t)$ dependence of the transition probability. The second term can average out to zero but the classical transition probability can still be measured. This is true for most of the so-called oscillation experiments which therefore should be named differently. The opposite special case occurs when coherence is exactly preserved or $x=t=0$ which gives unity for the exponential term. In this case $P(\alpha \rightarrow$ $\beta)=\delta_{\alpha \beta}$ and no transitions are possible.

In realistic neutrino oscillation experiments the neutrino masses are probed in the sub$\mathrm{keV}$ region whereas experimentally accessible neutrino momenta are in the $\mathrm{MeV}$ and $\mathrm{GeV}$ range. Therefore the simplifying assumption of ultra-relativistic neutrinos is valid. It can be expressed in the following way:

$$
\begin{aligned}
& E_{i}=E_{j} \equiv E ; \quad \forall i, j \\
& p_{i}=E-m_{i}^{2} / 2 E
\end{aligned}
$$

The choice of assuming equal energies and different momenta is arbitrary. The opposite assumption

$$
\begin{aligned}
& p_{i}=p_{j} \equiv p ; \quad \forall i, j \\
& E_{i}=p+m_{i}^{2} / 2 p .
\end{aligned}
$$

leads to exactly the same result apart from the detail that one has to replace the average energy $E$ by the average momentum $p$ in the final formula which is insignificant in the ultra-relativistic limit. With the above approximation the transition probability simplifies to:

$$
P(\alpha \rightarrow \beta)=\sum_{i}\left|U_{\beta i}\right|^{2}\left|U_{\alpha i}\right|^{2}+R e \sum_{i \neq j} U_{\beta i} U_{\beta j}^{*} U_{\alpha i}^{*} U_{\alpha j} \exp ^{-i t \Delta m_{i j}^{2} / 2 E}
$$


with

$$
\Delta m_{i j}^{2}=\left|m_{i}^{2}-m_{j}^{2}\right|
$$

Up to now we did not use any specific properties of neutrinos for the evaluation of the transition probability. The starting point was to copy of the quark mixing scheme into the lepton sector. Therefore the result should hold as well for the quark sector assuming $U_{\alpha i}$ the quark mixing matrix. Is it possible to perform "Quark oscillation experiments" as well?

Neglecting the fact that quarks are not observable as free particles due to confinement, we can treat the quarks analogously to the neutrinos. From the phase term we can extract the oscillation wave length

$$
\lambda_{i j}=4 \pi E / \Delta m_{i j}^{2}
$$

To maximise the oscillation wave length for the quark sector assume $p=1 \mathrm{TeV}$ and $\Delta m_{i j}^{2}=0.01 \mathrm{GeV}^{2}$ (which is the minimal possible value, taking $\mathrm{d}$ and $\mathrm{s}$ quarks). This leads to an oscillation wavelength of

$$
\lambda_{d s}=2.4 \cdot 10^{-10} \mathrm{~m},
$$

which is unobservable. This means for the quark sector the quantum-mechanical phase information is not accessible to experiments. One measures just the classical transition probability. This is done, e.g. in flavour changing weak decays.

For neutrinos the situation is different due to the different mass scale of interest. Assuming $p=1 \mathrm{MeV}$ and $\Delta m_{i j}^{2}=1 \mathrm{eV}^{2}$, the oscillation wave length is

$$
\lambda_{\nu}=2.4 m
$$

which is a macroscopic value which could by tested by a suitable experiment. Nevertheless if the phase information cannot be measured in a neutrino oscillation experiment the result would be still of great interest because up to now transition processes between different lepton flavour families have never been observed.

Based on the knowledge that (at least) three lepton families exist, results of oscillation experiments would have to be expressed in a 6-parameter space:

- $\Delta m_{12}^{2}, \Delta m_{13}^{2}$

- $U_{\alpha i}$ three mixing angles and one CP-violating phase.

The three squared mass differences are linearly dependent, so one has only two free squared mass difference scales:

$$
\Delta m_{12}^{2}+\Delta m_{23}^{2}=\Delta m_{13}^{2} .
$$

However most experiments present their results in a two flavour approximation, which assumes that one $\Delta m^{2}$ scale and one mixing angle dominate the effect. The mixing matrix becomes simply

$$
U_{\alpha i}=\left(\begin{array}{rr}
\cos \theta & \sin \theta \\
-\sin \theta & \cos \theta
\end{array}\right),
$$

and a CP-violating phase cannot be accomodated. The transition probability simplifies to

$$
P(\alpha \rightarrow \beta)=\left|\delta_{\alpha \beta}-\sin ^{2} 2 \theta \sin ^{2}(\pi L / \lambda)\right|
$$

with $L=c t$ the distance from neutrino source to detection point. Each oscillation experiment is characterized by the following parameters: 
- The minimal transition probability $P_{\min }$ between two different weak eigenstates $\alpha$ and $\beta$ which can be detected (for experiments measuring $P(\alpha \rightarrow \alpha)$ the relation $P(\alpha \rightarrow \beta)=1-P(\alpha \rightarrow \alpha)$ holds in the two-flavour approximation)

- the mean neutrino energy $\mathrm{E}$

- the mean distance between source and detector L

- the "width" of the L/E distribution

Results are presented in a two-parameter space of $\sin ^{2} 2 \theta$ and $\Delta m^{2}$. Figure 4 indicates how the experiment-related parameters $P_{m i n}, \mathrm{~L}$ and $\mathrm{E}$ and the oscillation-related parameters $\sin ^{2} 2 \theta$ and $\Delta m^{2}$ are correlated.

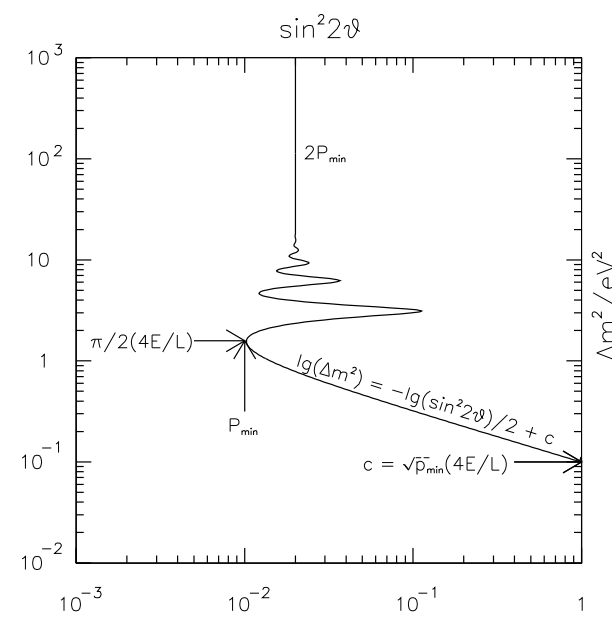

Figure 4: Sensitivity of a typical neutrino oscillation experiment; minimal transition probability $1 \%$; mean value of $4 E / L=1 \mathrm{eV}^{2}$ (e.g. neutrino energy of $1.27 \mathrm{GeV}$ and distance source-detector of $1 \mathrm{~km}$ ); width of the $\mathrm{L} / \mathrm{E}$ distribution : $10 \%$ of the mean value for $\mathrm{L} / \mathrm{E}$ (assuming a Gaussian L/E distribution)

- For small $\Delta m^{2}$, corresponding to $\lambda \gg L$, the phase term $\sin ^{2}(\pi L / \lambda)$ can be approximated by $(\pi L / \lambda)^{2}$. This results in a straight line of experiment-independent slope in a double-logarithmic plot such as in figure 4.

- The value of $\Delta m^{2}$, for which the sensitivity in $\sin ^{2} 2 \theta$ is maximal, is reached for $L=\lambda / 2$ independent of $P_{\min }$.

- For large $\Delta m^{2}$, corresponding to a small oscillation length, the phase information is lost due to the necessary convolution with the $\mathrm{L} / \mathrm{E}$ distribution. It remains the classical transition probability

$$
P(\alpha \rightarrow \beta)=\left|\delta_{\alpha \beta}-\frac{1}{2} \sin ^{2} 2 \theta\right| .
$$

In case of a negative result (no oscillation signal observed) the parameter region on the right side of the curve, given in figure 4, would be excluded. Observation of an oscillation signal would instead result in a preferred parameter region within a band along this curve. 
Lepton flavour violating weak decays can be interpreted in the framework of neutrino oscillations as well. A popular example is the decay $\mu \rightarrow e \gamma$. This decay can be expressed by Figure 3 by combining the two W-lines into an internal loop. To complete the diagram a photon has to be added at one of the charged particle lines. The partial decay width $\Gamma(\mu \rightarrow e \gamma)$ is proportional to the oscillation probability $P\left(\nu_{\mu} \rightarrow \nu_{e}\right)$. The term E/L, related to the oscillation phase, has to be replaced by $m_{W}^{2}$, because the lifetime of the quantum fluctuation, which consists of the W-boson and the $\nu_{i}$, is dominated by the heavy W-boson. Therefore the approximation for small $\Delta m^{2}$ can be applied and considering two neutrino flavours Eq. 30 modifies to

$$
\Gamma(\mu \rightarrow e \gamma) \propto P\left(\nu_{\mu} \rightarrow \nu_{e}\right) \approx \sin ^{2} 2 \theta \frac{\left(\Delta m^{2}\right)^{2}}{16 m_{W}^{4}} .
$$

For $\Delta m^{2}=1 \mathrm{MeV}^{2}$ one obtains a suppression for this decay of the order of $10^{-20}$ which is much stronger than the best experimental limits.

From the experimental point of view the following techniques of oscillation experiments can be distinguished:

Appearance experiments try to detect a neutrino flavour $\beta \neq \alpha$ in some distance from a neutrino source, where mainly $\nu_{\alpha}$ are created. The fraction of prompt $\nu_{\beta}$ should be small. One measures directly $P(\alpha \rightarrow \beta)$ where $\alpha$ stands for all flavours which are created in the source which much larger abundance than $\nu_{\beta}$. The beam energies must be high enough to allow the creation of $l_{\beta}$ (the charged lepton of flavour $\beta$ ).

Disappearance experiments try to measure deviations from the expected flux of one neutrino flavour at the detector position. These experiments are useful, because if the neutrino energy is below the kinematical threshold of producing $l_{\beta}$ it is impossible to perform an appearance experiment. Solar and reactor neutrino experiments are in this situation with neutrino energies in the $\mathrm{MeV}$ range $(\alpha=e, \beta=\mu, \tau)$. The measured sensitivity in $(1-P(\alpha \rightarrow \alpha))$ can be interpreted as $P(\alpha \rightarrow \beta)$ for arbitrary $\beta$ (including exotic flavours such as sterile neutrinos).

Two-detector disappearance experiments compare the measured neutrino flux of flavour $\alpha$ at two different distances from the source. The sensitivity in $\Delta m^{2}$ is limited to a small range corresponding to the condition $L_{1}<\lambda<L_{2}$ with $L_{1}$ and $L_{2}$ denoting the distances of the near and far detectors from the neutrino source.

Other methods are mixtures of appearance and disappearance technology. Examples are described in details in sections 4.4 and 4.6.2.

The results of different experiments will be compared in three different channels $\nu_{e} \leftrightarrow$ $\nu_{\mu}, \nu_{e} \leftrightarrow \nu_{\tau}$ and $\nu_{\mu} \leftrightarrow \nu_{\tau}$. No distinction will be made between $\nu_{\alpha} \rightarrow \nu_{\beta}$ and $\nu_{\beta} \rightarrow \nu_{\alpha}$ which means neglecting CP-violating effects which could occur in a three flavour scheme. Furthermore, there will be no distinction between results from neutrino and antineutrino channels.

\subsection{Matter-enhanced oscillations}

The approach, used in the last section, assumes that the neutrino mass eigenstates propagate in vacuum. For solar neutrinos, which are produced in the core of the Sun, matter 
effects inside the Sun (or even inside the Earth) could modify the detectable oscillation pattern and have to be taken into account.

When neutrinos pass through matter they undergo multiple small angle scattering processes which can be expressed in terms of a refraction index. For elastic scattering with nuclei the refraction indices are flavour independent because only Z-boson exchange contributes. For the scattering process $\nu+e \rightarrow \nu+e$ the refractive index will be different for (anti)electron neutrinos and other flavours due to the additional contribution of W-boson exchange in the scattering process. This difference in neutrino refraction indices will introduce an additional phase shift which can change the oscillation probability significantly. This concept was introduced and developed by Mikhejev, Smirnov and Wolfenstein [21] (MSW).

In the following we will restrict on the two-flavour approximation in the limit of small oscillation wavelengths. Therefore averaging over the phase term has been done. So the starting point is Eq. 31 which modifies for a material of constant electron density $n_{e}$ to

$$
P\left(\nu_{e} \rightarrow \nu_{e}\right)=1-\frac{1}{2} \sin ^{2} 2 \theta_{m}=\frac{1}{2}+\frac{1}{2} \cos ^{2} 2 \theta_{m},
$$

where the index $\mathrm{m}$ indicates "matter". The relation to the vacuum formula is given by

$$
\begin{array}{ll}
\sin 2 \theta_{m} & =\sin (2 \theta) / R \\
\cos 2 \theta_{m} & =\left(\cos (2 \theta)-A / \Delta m^{2}\right) / R \\
R & =\sqrt{\left(A / \Delta m^{2}-\cos 2 \theta\right)^{2}+(\sin 2 \theta)^{2}} \\
A & =2 \sqrt{2} G_{F} n_{e} E .
\end{array}
$$

where $G_{F}$ is the Fermi constant, $n_{e}$ the electron density and $\mathrm{E}$ is the neutrino energy. For $n_{e}=0$ one obtains $A=0$ and $R=1$, which reduces the effect to that of vacuum oscillations. A resonance occurs for

$$
A=\Delta m^{2} \cos 2 \theta
$$

where the effective mixing becomes maximal, i.e. $\sin ^{2} \theta_{m}=1$. The resonance disappears if one of the following changes is applied:

- $\bar{\nu}_{e}$ are considered instead of $\nu_{e}\left(n_{e} \rightarrow-n_{e}\right)$

- the mass hierarchy of the 2 neutrinos is inverted $(\theta \rightarrow \pi / 2-\theta)$

Performing both changes simultaneously (antineutrinos and a $\bar{\nu}_{e}$, which couples stronger to the heavier mass eigenstate) the resonance remains.

The Sun is not a medium of fixed density. One can describe the Sun's density with an exponential behaviour starting with a maximal value in the centre and decreasing smoothly up to vacuum value. If the density change is small enough to fulfill the condition

$$
\left|d \theta_{m} / d x\right| \ll \pi R / \lambda
$$

the neutrinos propagate many oscillation wavelengths under quasi fixed-density conditions. This is called adiabatic approximation. In general, one can give the oscillation probability for a complete adiabatic transition between two regions of different density $(m 1$ and $m 2)$ by modifying Eq. 33 to

$$
P\left(\nu_{e} \rightarrow \nu_{e}\right)=\frac{1}{2}+\frac{1}{2} \cos 2 \theta_{m 1} \cos 2 \theta_{m 2} .
$$


It is easy to verify that $P\left(\nu_{e} \rightarrow \nu_{e}\right) \rightarrow 0$ if the transition occurs between a medium with a density much larger than the resonant density $\left(\theta_{m 1}=\pi / 2\right)$ and vacuum with a small mixing angle $\left(\theta_{m 2} \approx 0\right)$, so the resonance density has been crossed. For vacuum oscillations one can reach a maximal suppression of $50 \%$ of the initial flux in the case where the phase information has been lost and the two flavour approximation is used. This important difference will be used in the discussion of the experimental results.

To get a complete description one has finally to take into account that the adiabatic approximation can break down, which happens when the oscillation wave length gets too large to fulfill Eq. 36. The breakdown of the adiabatic condition can be described by a complicated function $F$ and Eq. 37 modifies to:

$$
P\left(\nu_{e} \rightarrow \nu_{e}\right)=\frac{1}{2}+\left(\frac{1}{2}-F\right) \cos 2 \theta_{m 1} \cos 2 \theta_{m 2}
$$

For simplicity we only consider the limiting cases. If the adiabatic approximation is fulfilled $F=0$, whereas for its complete failure one obtains $F=\cos ^{2} \theta$, which reduces Eq. 38 to the vacuum oscillation formula (together with $\theta_{m 1}=\pi / 2, \theta_{m 2}=\theta$ ).

The triangular shape of the MSW contours is shown in figure 5 . It can be understood in

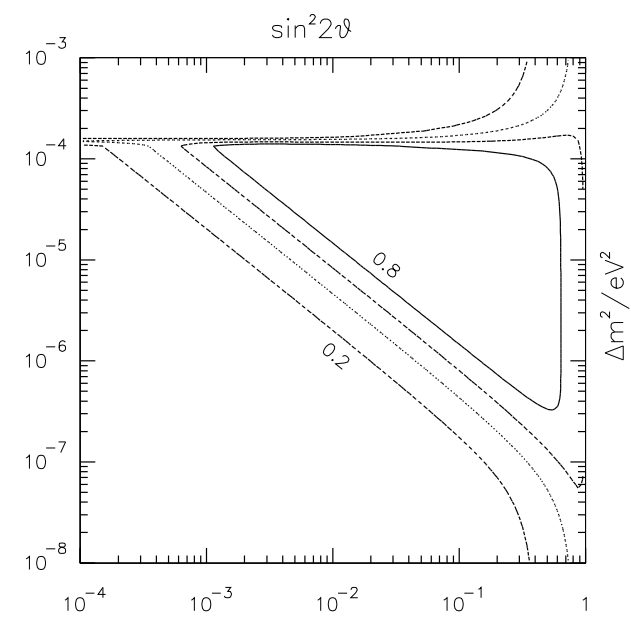

Figure 5: MSW effect for $10 \mathrm{MeV}$ solar neutrinos. The lines show $\left(1-P\left(\nu_{e} \rightarrow \nu_{e}\right)\right)=$ $0.2,0.4,0.6,0.8$.

the following way: Every value of $\Delta m^{2}$ refers to a specific resonant density (see Eq.35). For high values of $\Delta m^{2}$ the resonance density cannot be reached and matter enhancement does not occur. Decreasing $\Delta m^{2}$ one reaches a regime where the resonant density is the core density of the Sun. This point defines the horizontal line of the triangle. Further reduction of $\Delta m^{2}$ moves the resonant density away from the core but the adiabatic approximation is still valid. This holds for the inner part of the triangle where practically all neutrinos are turned into a different flavour. A further decrease of $\Delta m^{2}$ will make the adiabatic approximation invalid, depending on $\sin ^{2} 2 \theta$, which defines the inclined line of the triangle. If one moves from the inner part of the triangle in horizontal direction towards a large mixing angle than the size of the resonance becomes smaller (see again Eq.35) until it completely disappears for maximal mixing.

An extensive discussion of the MSW effects and a detailed derivation of all formulas given in this section can be found, e.g., in [22]. 


\subsection{Solar neutrinos}

The Sun supplies the highest flux of neutrinos on Earth with about $6 \cdot 10^{10}$ neutrinos per $\mathrm{cm}^{2} \mathrm{~s}$. Solar neutrinos are produced by the nuclear fusion processes in the core of the Sun. The most important processes supplying neutrinos are generated in the so-called pp-cycle:

$$
\begin{array}{lll}
p+p & \rightarrow{ }_{1}^{2} D+e^{+}+\nu_{e} & 0.42 \mathrm{MeV} \\
p+p+e^{-} & \rightarrow{ }_{1}^{2} D+\nu_{e} & 1.44 \mathrm{MeV} \\
7{ }_{4} B e+e^{-} & \rightarrow{ }_{3}^{7} L i+\gamma+\nu_{e} & 0.86 \mathrm{MeV} \\
{ }_{5}^{8} B & \rightarrow 2 \alpha+e^{+}+\nu_{e} & 14.6 \mathrm{MeV} .
\end{array}
$$

The given energies are the neutrino energies for the electron capture reactions and the maximal neutrino energies for the beta decay reactions. All reactions in the Sun produce electron neutrinos which can be understood looking at the net reaction of the pp-cycle:

$$
2 e^{-}+4 p \rightarrow{ }^{4} \mathrm{He}+2 \nu_{e} \quad 26.7 \mathrm{MeV} .
$$

The spectra of the neutrino fluxes from the various channels (including as well neutrinos from the less significant CNO cycle) are given in figure 6 [23]. The fundamental pp reaction is closely related to the overall luminosity of the Sun and can be precisely predicted. The Be and $\mathrm{B}$ reactions represent only a small fraction of the overall cycle and the branching ratios depend strongly on various parameters of the solar model, such as the temperature of the core of the Sun, various nuclear cross sections etc. The only well established correlation between the $\mathrm{Be}$ and $\mathrm{B}$ flux comes from the fact that $\mathrm{B}$ is made from $\mathrm{Be}$ and every modification to the Be flux would affect the B-neutrinos in the same way.

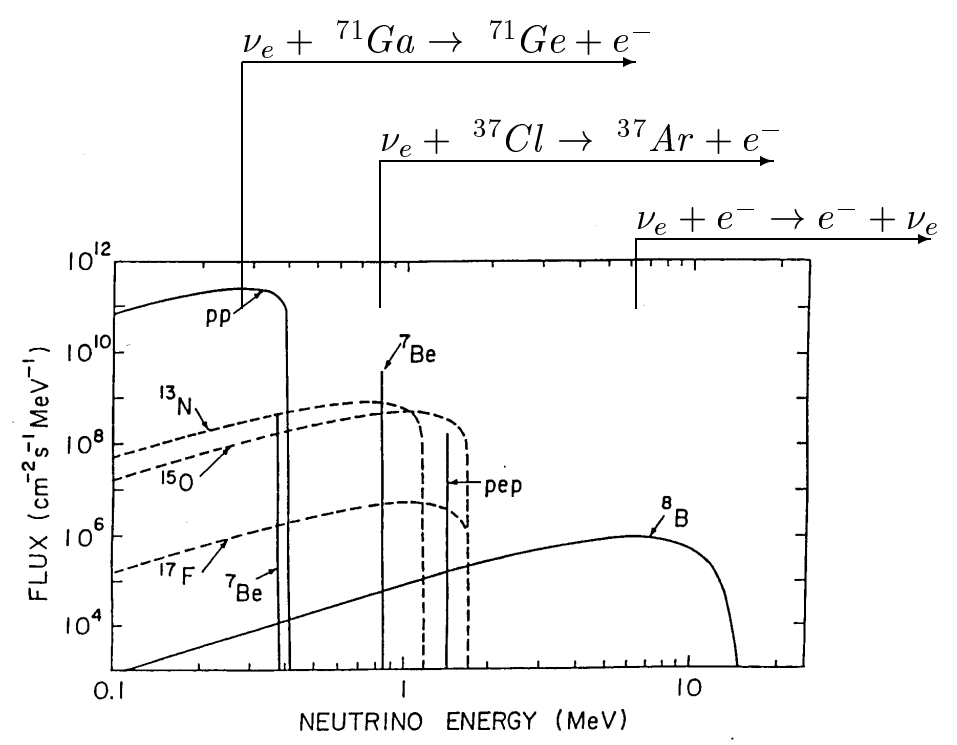

Figure 6: Components of the solar neutrino flux and detection thresholds for the different reactions used in the solar $\nu$ experiments [23]

\section{Radiochemical experiments}

To test the pp-branch and Be-branch of the spectrum one has to find a technique which allows a detection of neutrinos with energies below $1 \mathrm{MeV}$. This can be done by choosing 
a beta decay or electron capture process with an energy gap between the two nuclei below the threshold value of the process of interest. The solar neutrinos could then interact via inverse beta decay and create the heavier beta-instable nucleus which subsequently will be counted. Only a few atoms within many tons of target material will be created within weeks by reactions of the solar neutrinos but the counting of these atoms becomes possible by observing their beta decay (or the gammas related to the electron capture reaction). Therefore the experiments operate in cycles. An exposure period is followed by an extraction and counting period. Both scales are typically weeks. The only information which can be extracted from radiochemical experiments is therefore the number of events above threshold for an exposure period. This means the following information cannot be deduced from radiochemical experiments:

- neutrino energy spectrum

- directional correlation of events and Sun

- short-term time dependencies such as day/night fluctuations

However long-term time dependencies such as seasonal fluctuations can be studied.

Gallium experiments Two experiments, GALLEX and SAGE, are running to study the pp-branch of the solar spectrum using the inverse beta decay of ${ }^{71} G a[24,25]$

$$
{ }_{31}^{71} G a+\nu_{e} \rightarrow{ }_{32}^{71} G e+e^{-} ; E_{t h r}=0.233 \mathrm{MeV} .
$$

The produced Germanium has a half-life of 16.5 days. Therefore the exposure period is one month followed by a counting period of several months. According to [23] the different branches of the spectrum should contribute in the following way:

$$
137 \pm 8=70(p p)+38\left({ }^{7} \mathrm{Be}\right)+16\left({ }^{8} \mathrm{~B}\right)+10(\mathrm{CNO})+3(\text { pep })
$$

The numbers are given in SNU (1 SNU is 1 capture per second per $10^{36}$ target atoms). This has to be compared with the measured rate of $[24,25]$

$$
\begin{array}{lll}
\text { GALLEX } & 70 \pm 8 & \text { SNU } \\
\text { SAGE } & 72 \pm 12 \pm 7 & \text { SNU }
\end{array}
$$

which refers to $51 \%$ (53\%) of the expected flux. The GALLEX result refers to fully analysed data taken from 1991 to 1995, whereas for SAGE the result describes the data taken in the period 1990 till 1993.

To prove the reliability of their counting method both experiments performed a calibration with a radioactive ${ }^{51} \mathrm{Cr}$ source. The source was activated at a nuclear reactor. The ${ }^{51} \mathrm{Cr}$ isotope has a half-life of 27 days and $90 \%$ of the neutrinos produced have an energy of $746 \mathrm{keV}$, so they sample well the energy range for the solar pp and Be neutrinos. The activity of the source could be determined by various methods (neutron flux measurements, calorimetry, spectroscopy) to $1 \%$ precision. The final results for the ratio of the measured to expected neutrino flux from the ${ }^{51} \mathrm{Cr}$ source is given as $[24,25]$

$$
\begin{array}{ll}
\text { GALLEX } & 0.92 \pm 0.07 \\
\text { SAGE } & 0.95 \pm 0.11 \pm 0.07
\end{array}
$$

This means that unknown systematic effects which could cause a lower count rate of solar neutrinos have to be smaller than about $10 \%$. 
Chlorine experiment The pioneering solar neutrino experiment, Homestake, [26] has been recording solar neutrinos for more than 20 years. It works with a different isotope, looking for the reaction

$$
{ }_{17}^{37} \mathrm{Cl}+\nu_{e} \rightarrow{ }_{18}^{37} \mathrm{Ar}+e^{-} ; E_{t h r}=0.813 \mathrm{MeV}
$$

The threshold energy is just below the energy for the Be branch, so the expected flux comes mainly from Be and B as it is shown in the predictions from [23] :

$$
9.3 \pm 1.3=0.0(p p)+1.2\left({ }^{7} B e\right)+7.4\left({ }^{8} B\right)+0.5(C N O)+0.2(p e p),
$$

where the numbers are again in SNU. The measured flux is based on data taken between 1970 and 1994 [26]:

$$
\text { Homestake } \quad 2.54 \pm 0.16 \pm 0.14 \quad \text { SNU }
$$

The discrepancy between measurement and prediction is here even more pronounced. Only $28 \%$ of the expected flux can be found. The Homestake experiment has never been calibrated with an independent strong radioactive source as it has been done for the two Gallium experiments.

\section{Water Čerenkov experiments}

The basic principle of these experiments is to look for neutrino electron scattering processes of the type

$$
\nu+e^{-} \rightarrow \nu+e^{-}
$$

and to detect the Čerenkov light of the scattered electron. There is no kinematical threshold for this reaction but background problems (Čerenkov light from radioactivity and cosmic-ray particles) require a threshold of a few $\mathrm{MeV}$ (typically 5-7 MeV). Therefore these experiments are only sensitive to neutrinos from the B branch of the solar neutrino flux.

Another important feature comes from the universal character of neutrino electron scattering. This process is possible for all types of (anti)neutrinos, but with different cross sections :

$$
\sigma\left(\nu_{e} e^{-}\right): \sigma\left(\nu_{\mu, \tau} e^{-}\right): \sigma\left(\bar{\nu}_{e} e^{-}\right): \sigma\left(\bar{\nu}_{\mu, \tau} e^{-}\right)=1: 0.16: 0.41: 0.14
$$

This means even in case of complete oscillation a signal of at least $16 \%$ of the expected flux must be seen; otherwise an interpretation in the frame of neutrino oscillations (without considering exotic phenomena such as sterile neutrinos) becomes impossible. The correlation between the ratio $R=$ "measured flux/expected flux" and the neutrino oscillation probability $P\left(\nu_{e} \rightarrow \nu_{e}\right)$ is given by

$$
P\left(\nu_{e} \rightarrow \nu_{e}\right)=(R-0.16) /(1-0.16) .
$$

In contrast to the radiochemical experiments the detection of Cerenkov light is done in real time. This means short time variations such as day/night effects or neutrino bursts (e.g. from supernovae) could be seen. If the energy of the recoil electron is much larger than the electron mass $\left(E_{e} \gg m_{e}\right)$ (which is always fulfilled due to the detection threshold 
of at least $5 \mathrm{MeV}$ ) the angle of the electron with respect to the incoming neutrino $(\theta)$ is limited to values

$$
\theta \quad \sqrt{2 m_{e} / E_{e}}
$$

Therefore a good angular correlation between the signal and the direction of the Sun can be obtained. Also spectral information can be deduced from the Cerenkov light but here the correlation is weaker by the flat $y$-distribution $\left(y=E_{e} / E_{\nu}\right)$ of $\nu_{e} e^{-}$scattering.

Kamiokande The Kamiokande experiment [27] consisted of a 4500-ton water tank covered by 948 photo multipliers. A fiducial volume of 680 tons was used to detect solar neutrinos. The experiment took data from December 1986 until April 1996 with more than 2000 days of active data taking. An energy threshold of $7 \mathrm{MeV}$ was used. For a $10 \mathrm{MeV}$ signal an energy resolution of $19 \%$ and an angular resolution of $28^{\circ}$ has been measured. An event rate of 6 events per day could be obtained which (after taking into account the directional correlation with the Sun) gives a neutrino flux of

$$
2.80 \pm 0.19 \pm 0.33 \cdot 10^{6} \mathrm{~cm}^{-2} \mathrm{~s}^{-1}
$$

which is $42 \%$ of the expected value according to [23]. Assuming neutrino oscillations and applying Eq. 50 this value corresponds to an oscillation probability $P\left(\nu_{e} \rightarrow \nu_{e}\right)$ of $31 \%$, which is compatible to the Homestake result.

SuperKamiokande In April 1996 an upgraded version of Kamiokande started data taking. It consists of a 50-kiloton water tank surrounded by 11,200 photo multipliers. First results from 28 days of data taking were presented [28]. A fiducial volume of 11.7 kilotons is used for this preliminary analysis and an energy threshold of $7 \mathrm{MeV}$. A signal of $182 \pm 25$ solar neutrino events $(0.56 \pm 0.08$ events per day per kiloton) has been observed which is comparable to the total dataset of Kamiokande. The normalisation in terms of solar neutrino flux has still to be done.

Superkamiokande is expected to supply a 100 times higher rate of solar neutrino events than Kamiokande. This is due to the much larger target mass and a reduced energy threshold which is expected to be at $5 \mathrm{MeV}$ for Superkamiokande .

\section{Interpretation of results}

All solar neutrino experiments see a deficit of the measured flux compared to solar models. Combining the results of all experiments, with their different energy thresholds, there seems to be a spectral dependence of the deficit. Time variations of the signal are not observed. The anticorrelation of the neutrino flux and the sunspot number, originally found by Homestake [29], could not be confirmed by any of the other experiments. [24, $25,27]$ A reanalysis of the Homestake data showed that the data are well described by a time constant neutrino flux [26]. In the following, various options are discussed, which have been proposed to explain the data. For every option, a specific way is shown, which could help to distinguish it from other possibilities.

Solar Model modification The shape of the different contributions to the solar neutrino spectrum is known with a precision better than $1 \%$ [30], which is much more precise than the relative normalisation of the branches. Therefore a minimal assumption about the neutrino-producing processes in the Sun is to preserve the shapes of 


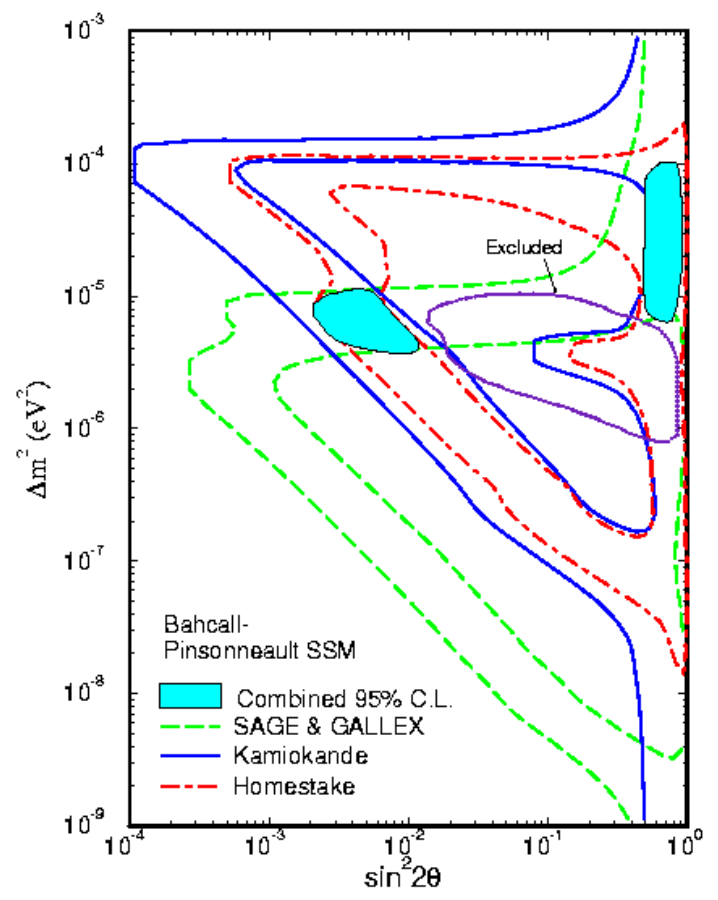

Figure 7: allowed regions of oscillation parameters for resonant amplification of the oscillation (MSW effect) [33]

the different branches but assume arbitrary relative normalisation with respect to each other. These relative normalisations can be determined by performing a fit to the experimental data. This has been done and the data are inconsistent with the above minimal assumption at the $94 \%$ C.L. Adding the luminosity constraint, the inconsistency becomes $99.5 \%$ C.L. [31]. This approach does not include detailed assumptions about the temperature of the core of the Sun, nuclear cross sections etc. Adding further constraints, the disagreement between data and solar model becomes even more evident. From the experimental point of view the detection of neutral-current (NC) reactions could distinguish an oscillation from an astrophysical solution of the solar neutrino deficit. The rate of $\mathrm{NC}$ interactions should be independent from any oscillation pattern because NC reactions are "flavour blind" (with the exception of sterile neutrinos).

vacuum oscillations To account for the Homestake result with its dramatic suppression of the neutrino flux one has to tune the oscillation wavelength to be of the order of the Sun-Earth distance. For a $1 \mathrm{MeV}$ neutrino the first maximum occurs at $\Delta m^{2} \approx 10^{-11} \mathrm{eV}^{2}$. Without oscillations one expects a $7 \%$ variation of the solar flux between perihelion and aphelion of the Earth's orbit. This effect is too small to be detectable in the present results. If the oscillation wave length $\lambda$ is of the order of the Sun-Earth distance, $x$, one would expect a different (not necessarily larger !) value according to the different contribution of $\sin ^{2}(\pi x / \lambda)$. Future experiments can verify the vacuum oscillation hypothesis by observing seasonal variations of the neutrino flux.

vacuum oscillation - maximal mixing The two gallium experiments are compatible with the assumption of $50 \%$ reduction of the flux. This can be reached assuming 
vacuum oscillations in a two flavour approximation (Eq. 31), where the phase information has been lost. In this case the solar neutrino deficit would not contribute in fixing a scale for $\Delta m^{2}$ and can be combined with other positive results. This approach has been proposed e.g. in [32]. However one has to assume that the Homestake and Kamiokande results or their flux predictions or both are wrong by at least 4 standard deviations. In future precise measurements this possibility would manifest itself as an energy-independent suppression of the complete neutrino spectrum without any time variations (apart from the $7 \%$ orbit effect mentioned above).

matter-enhanced oscillations The matter enhancement of the oscillation probability gives a possibility of reaching large suppression factors due to the resonance effect. Due to the different energies which are tested in the various experiments one observes a complex pattern of preferred oscillation parameters as shown in figure 7 [33]. Two regions can be identified which are consistent with all experimental results. One is normally referred to as "small-angle solution". It occurs for a resonance density which is close to the density of the solar core for pp neutrinos (which are measured in the Gallium experiments) and a resonance density close to the breakdown of the adiabatic solution for $\mathrm{B}$ neutrinos which give the main contribution of the remaining experiments. The second consistency region, the so-called "large-angle solution", is compatible to the "maximal mixing" discussion, but here the Homestake result limits the validity of the $\Delta m^{2}$ range. The MSW-solution predicts strong spectral distortions of the solar neutrino flux. A precise differential measurement of the energy dependence of the solar neutrino flux would test this hypothesis. An additional region of exclusion, diminishing the allowed region for the large angle solution, can be derived from the non-observation of day/night fluctuations in Kamiokande, which means the absence of strong matter-enhanced effects inside the Earth. Further observations of day/night variations of the solar neutrino flux will help to distinguish between the "small-angle" and "large-angle" solution. For the latter a more pronounced day/night effect is predicted.

\section{Future projects}

Unfortunately results of solar neutrino experiments cannot be tested with neutrino sources available on Earth. To reach $\Delta m^{2} \approx 10^{-5} \mathrm{eV}^{2}$ one would need a baseline of at least 1000 $\mathrm{km}$ for $10 \mathrm{MeV}$ neutrinos. Nuclear reactors are the strongest source of (anti)neutrinos in this energy range but the most challenging proposals for experiments at reactors consider baselines up to $10 \mathrm{~km}$.

There are several future solar neutrino experiments planned, some of them already at the construction phase.

SNO This experiment [34] works with Čerenkov light as does Kamiokande but it uses heavy water $\left(D_{2} O\right)$. Therefore new reaction channels for neutrinos open:

$$
\begin{array}{lllll}
\mathrm{CC} & \nu_{e}+D & \rightarrow p+p+e^{-} & 1.4 \mathrm{MeV} & (1) \\
\mathrm{NC} & \nu+D & \rightarrow p+n+\nu & 2.2 \mathrm{MeV} & (2) \\
\mathrm{CC} & \bar{\nu}_{e}+D & \rightarrow n+n+e^{+} & 4.0 \mathrm{MeV} & (3)
\end{array}
$$

where the energy denotes the threshold for this process. An unique possibility is the measurement of neutral-current reactions. This could distinguish astrophysical explanations 
(which would change the overall neutrino flux) from neutrino oscillation solutions (which would leave the flavour blind neutral-current processes unchanged). The signal for the neutral-current reactions is a single neutron whose detection and separation from background is extremely difficult. Neutrons can only be detected by the photons related to their capture. Various nuclei have been considered for SNO to enhance the capture rate and increase the photon energies. No final decision has been taken so far.

A total mass of 1000 tons of $D_{2} O$ is foreseen. For an energy threshold of $6 \mathrm{MeV}$ one expects 6800 charge-current events and 2000 neutral-current events per year, assuming a flux of the B neutrinos according to the solar standard model. This can be compared with 680 expected events from neutrino electron scattering, the only available channel for other water Čerenkov detectors.

There are major differences in energy and angular resolution for reaction (1) and neutrino-electron scattering. Whereas for $\nu e$ scattering the angular resolution for the neutrino direction is good (Eq. 51), one obtains only a $\left(1-\frac{1}{3} \cos \theta\right)$ asymmetry for reaction (1) $(\theta$ denotes the angle of the electron with respect to the incoming neutrino). For the neutrino energy resolution one expects less significant results for $\nu e$ scattering due to the flat $\mathrm{y}$-distribution of this process, whereas the electron in the deuteron chargedcurrent process receives almost all the neutrino energy because of the limit $E_{\nu} \ll m_{D}, m_{p}$. Therefore spectral distortions should be measured more precisely here. The schedule anticipates filling the detector with $D_{2} \mathrm{O}$ in 1997.

Borexino The basic idea of this experiment [35] is to record scintillation light from $\nu-e$ scattering. The detector consists of a $8.5-\mathrm{m}$ diameter transparent nylon inner vessel filled with 300 tons of liquid scintillator and viewed by about 2000 photomultiplier tubes. Scintillation light detection allows a higher energy resolution compared to Čerenkov light determination but the background suppression is more complicated, especially if one wants to work with a very low energy threshold $(250 \mathrm{keV})$ as it is foreseen here. This allows the measurement of a completely differential solar neutrino spectrum (especially the Be part), only excluding the very low energy pp neutrinos. In this way Borexino would be very sensitive to small spectral distortions over a wide range of neutrino energies. The main problem in detection of scintillation signals in this energy range is background suppression. So the most challenging demands come from purity requirements of various components which are involved.

A test setup has been built to show the feasibility of the designed purity values. A sphere of 1 meter radius of 4.5 tons of liquid scintillator has been operated for 1 year in the Gran Sasso laboratory. Less than $10^{-15} \mathrm{~g}$ of Uranium and Thorium have been measured per gram of scintillator which fulfills the design goals of the experiment.

GNO The GNO project is designed to monitor the pp neutrino flux for a long time (at least 10 years) with a gallium detector at the Gran Sasso laboratory [36]. It has been approved in December 1996. The GNO upgrade will start after GALLEX has taken its last solar neutrino data in summer 1997. New electronics will be installed and first neutrino data can be taken in autumn 1997 with 30 tons of gallium (the GALLEX equivalent). An upgrade to 100 tons of gallium is foreseen within the next 5 years. Main objectives of GNO are a refinement of the measured solar neutrino capture rate at the gallium threshold and a search for long term time variations of the solar neutrino flux. 


\subsection{Atmospheric neutrinos}

When primary cosmic-ray particles (mainly protons and light nuclei) hit the upper part of the atmosphere they produce hadronic showers. Among them is a large fraction of pions.

$$
p+N \rightarrow \pi+X
$$

Due to the low density of the atmosphere most of the pions decay before interacting via

$$
\pi \rightarrow \mu+\bar{\nu}_{\mu} / \nu_{\mu}
$$

and a large fraction of the muons decays as well before reaching the ground

$$
\mu \rightarrow e+\bar{\nu}_{\mu} / \nu_{\mu}+\nu_{e} / \bar{\nu}_{e}
$$

From the above reaction scheme one would naively expect a ratio of (anti)muon neutrinos to (anti)electron neutrinos of two. Detailed simulations of the initial cosmic-ray spectrum and the subsequent shower development show that the absolute neutrino flux at a ground or underground detector can be predicted much less precisely than the flux ratio between the two neutrino flavours (see e.g. [37]). Therefore results which rely on absolute flux normalisation (such as measurements of the rate of upward going muons) are not taken into account here. Various experiments have measured the flux ratio between $\nu_{\mu}$ and $\nu_{e}$ but the results are generally given as double ratio

$$
R=\frac{\left(N\left(\nu_{\mu}\right) / N\left(\nu_{e}\right)\right)^{d a t a}}{\left(N\left(\nu_{\mu}\right) / N\left(\nu_{e}\right)\right)^{\text {simul }}} .
$$

If the simulation describes the data one would expect $R=1$. The energy range which is probed by these measurements covers roughly two orders of magnitude from a few $100 \mathrm{MeV}$ up to $10 \mathrm{GeV}$. Over this range the muon events are identified by the presence of a long minimum ionising particle. Some of the experiments analysed two data sets: so-called "fully contained" events, where interaction vertex and the stopping points of all tracks have to be inside the detector and "partially contained" events, which are instead characterised by an interaction vertex in the detector but a leaving particle (mostly the muon).

\section{Present status}

A comparison of the double ratio $R$ is shown for several experiments in table 1 .

\begin{tabular}{||c|c||}
\hline Experiment & $R$ \\
\hline Frejus [38] & $0.99 \pm 0.13 \pm 0.08$ \\
Nusex [39] & $1.04 \pm 0.25$ \\
Soudan2 [40] & $0.72 \pm 0.19 \pm 0.06$ \\
IMB [41] & $0.54 \pm 0.05 \pm 0.07$ \\
Kamiokande Sub-GeV [42] & $0.60 \pm 0.06$ \\
Kamiokande Multi-GeV [43] & $0.57 \pm 0.08 \pm 0.07$ \\
\hline
\end{tabular}

Table 1: Double ratio $R$ measured at different experiments 
The first three experiments are conventional tracking detectors whereas IMB and Kamiokande are water Cerenkov devices. The overall statistics are dominated by the water Čerenkov experiments. The disagreement between simulation and measurement is more pronounced for the latter class. Kamiokande also measured the zenith angle dependence of the double ratio for the multi-GeV events [43]. The deviation from unity is maximal for upward going events and it disappears for downward going events. A statistical significance larger than $99 \%$ for the non-flatness of the zenith angle distribution is quoted in [43]. However, the error propagation from the raw data to the "ratio of ratios" is questionable, as pointed out in [44]. A more precise handling of the statistical uncertainties reduces the significance to $94.8 \%$, which is less than two standard deviations [44].

Interpretation of the results of table 1 in terms of neutrino oscillations probes a mixing angle and mass difference range of

$$
\begin{array}{ll}
\sin ^{2} 2 \theta & >0.3 \\
\Delta m^{2} & >10^{-3} \mathrm{eV}^{2} .
\end{array}
$$

The deviation of $R$ from unity could be interpreted as $\nu_{\mu} \rightarrow \nu_{e}$ but as well $\nu_{\mu} \rightarrow \nu_{\tau}$ oscillations where the tau neutrinos do not contribute to charged-current reactions (they are below the energy threshold to produce charged tau leptons).

The interpretation of the total ratio $R$ in terms of oscillations gives just a lower limit for $\Delta m^{2}$ but the zenith angle dependence allows to fix a $\Delta m^{2}$ range. The observed dependence can be explained by assuming an oscillation wavelength smaller than the diameter of the Earth but larger than the typical flight path for downward going neutrinos. The best fit of the zenith angle dependence of the Kamiokande multi-GeV data [43] suggests maximal mixing $\left(\sin ^{2} 2 \theta=1\right)$ and

$$
\begin{array}{ll}
\Delta m^{2}=1.8 \cdot 10^{-2} \mathrm{eV}^{2} & \nu_{\mu} \rightarrow \nu_{e} \\
\Delta m^{2}=1.6 \cdot 10^{-2} \mathrm{eV}^{2} & \nu_{\mu} \rightarrow \nu_{\tau}
\end{array}
$$

For the sub-GeV data of Kamiokande no zenith angle dependence has been observed [43]. This does not contradict the oscillation hypothesis for the multi-GeV data, because the average oscillation wavelength is much shorter for sub-GeV data and therefore the averaging over the oscillation phase term becomes effective for a larger range of zenith angles.

Combining the Kamiokande results of the sub-GeV and multi-GeV data one obtains a preferred region of mixing parameters (90\% C.L.) for both oscillation hypotheses which is shown in figure 10. The strongest negative result for the same region of oscillation parameters, given by Frejus [38], has been included as well in figure 10 for comparison.

Some basic differences between the water Cerenkov experiments [41, 42, 43] and Frejus [38] are discussed in [38]:

- The experimental sites of Kamiokande and IMB are much more shallow than the one of Frejus, resulting in an atmospheric muon flux which is 20 times higher. Therefore the high energy neutron flux originating from muon interactions in the rock near the detector is expected to be higher.

- The analysis of water Cerenkov experiments is restricted to simple topologies (e.g. "single ring events"). In terms of neutrino physics this means a restriction to quasielastic events. However, the knowledge of quasi-elastic neutrino scattering is less 
advanced than the understanding of deep inelastic scattering. Frejus analysed the full data sample up to neutrino energies of $50 \mathrm{GeV}$. Quasi-elastic processes contribute only at a $30 \%$ level in the Frejus analysis.

\section{Future projects}

The next results for atmospheric neutrinos are expected to come from Superkamiokande, which uses the same methods as Kamiokande. Within 1997 new data for $R$ and the Zenith angle dependence for sub-GeV and multi-GeV data with more statistics can be expected.

The parameter space of neutrino mixing suggested by Kamiokande will be explored by future laboratory experiments as discussed in the following sections. They use "long baselines" to reach the required sensitivity in $\Delta m^{2}$. Here, "long baseline" means a distance between neutrino source and detector larger than $1 \mathrm{~km}$ for reactor experiments and distances larger than $100 \mathrm{~km}$ for accelerator experiments.

\subsection{Reactor neutrinos}

Nuclear reactors are an intense source of $\bar{\nu}_{e}$. Recall that the discovery of neutrinos was performed at a nuclear reactor [45]. The source of neutrinos are beta-decays related to the fission of heavy nuclei (mainly ${ }^{2} \mathrm{U},{ }^{239} \mathrm{Pu}$ ). Nuclear reactors produce exclusively $\bar{\nu}_{e}$, related to the net reaction $n \rightarrow p+e^{-}+\bar{\nu}_{e}$, which is necessary to create stable light nuclei. For nuclear fusion, as in the core of the Sun, the opposite reaction takes place leading to a flux of exclusively $\nu_{e}$.

\section{Present status}

A precise flux monitoring can be achieved by performing a

- determination of the thermal output of the reactor

- neutron flux measurement at the reactor

- reference measurements of the neutrino flux

A group working on the reactor at Rovno [46] has published an uncertainty for the total neutrino rate of $2.8 \%$.

The produced neutrinos have an energy of less than $8 \mathrm{MeV}$. The inverse beta decay is used to detect them:

$$
\bar{\nu}_{e}+p \rightarrow n+e^{+} \quad E_{\nu}>1.8 \mathrm{MeV} .
$$

The scintillation light of the positron or its annihilation photons is detected, followed by a signal from the neutron capture. For the neutron capture $\mathrm{Gd},{ }^{3} \mathrm{He}$ or ${ }^{6} \mathrm{Li}$ is used.

Due to the low neutrino energy, reactor neutrino oscillation searches are pure disappearance experiments, as solar neutrino experiments are. The results could be interpreted equally well as $\bar{\nu}_{e} \rightarrow \bar{\nu}_{\mu}$ or $\bar{\nu}_{e} \rightarrow \bar{\nu}_{\tau}$ oscillations. The sensitivity for the mixing angle is limited to values $\sin ^{2} 2 \theta>0.1$ due to the limited knowledge of the neutrino flux, whereas the sensitivity for $\Delta m^{2}$ varies considerably according to the experimental setup. Table 2 summarises the results of reactor experiments with the best current sensitivity in terms of oscillation parameters combined with two projects which are currently starting or scheduled for near future. The results of Bugey and Krasnojarsk are included into the general comparison of experimental results in figure 10. 


\begin{tabular}{||l|c|c|c|c||}
\hline Experiment & $\begin{array}{c}\text { target } \\
\text { mass }\end{array}$ & $\begin{array}{c}\text { reactor } \\
\text { distances }\end{array}$ & $\begin{array}{c}\Delta m^{2}\left(\mathrm{eV}^{2}\right) \\
\sin ^{2} 2 \theta=1\end{array}$ & $\begin{array}{c}\sin ^{2} 2 \theta \\
\Delta m^{2} \rightarrow \infty\end{array}$ \\
\hline Gösgen [47] & $320 \mathrm{~kg}$ & $38,46,65 \mathrm{~m}$ & $1.9 \cdot 10^{-2}$ & 0.21 \\
Krasnojarsk [48] & $600 \mathrm{~kg}$ & $57,230 \mathrm{~m}$ & $0.8 \cdot 10^{-2}$ & 0.15 \\
Bugey [49] & $600 \mathrm{~kg}$ & $15,40,95 \mathrm{~m}$ & $1.0 \cdot 10^{-2}$ & 0.15 \\
\hline Chooz [50] & $4,800 \mathrm{~kg}$ & $1025 \mathrm{~m}$ & $1.0 \cdot 10^{-3}$ & 0.1 \\
Palo Verde [51] & $12,000 \mathrm{~kg}$ & $750 \mathrm{~m}$ & $2.0 \cdot 10^{-3}$ & 0.1 \\
\hline
\end{tabular}

Table 2: Summary of reactor neutrino oscillation experiments (90\% C.L.). Above: results of past experiments ; Below : expected sensitivity of newly proposed experiments

\section{Future projects}

Comparing the new proposals with existing reactor experiments there is a clear tendency to use longer baselines to extend the sensitivity in $\Delta m^{2}$ by at least one order of magnitude. This is clearly motivated by the above-mentioned "atmospheric neutrino anomaly". The loss in flux (reactor neutrinos are produced isotropically, therefore the flux scales with $1 / r^{2}$ ) are compensated by larger detector masses (see table 2).

Chooz The experiment was completed in summer 1996. It has been installed in a cavern with 300 meter water-equivalent rock overburden which is an efficient shielding against cosmic-ray background. In September and October 1996 calibration data could be taken without the reactor running (which will start operation in early 1997). It could be shown that the background is below the estimated level [52]. The oscillation run is scheduled for two years with both reactors fully operational which should give a statistical error below $4 \%$. The sensitivity reached after the full running period is shown in figure 14 as an example for long baseline reactor experiments.

Palo Verde The excavation and construction of the underground lab $(25 \mathrm{~m}$ below ground) has been completed. The installation of the detector is in progress and data taking is expected to start in 1997.

Both experiments use an acrylic vessel filled with gadolinium-loaded scintillator as the core of the detector. The scintillation light from the positron and the neutron capture is recorded by photomultiplier tubes surrounding the vessel.

If the observed event ratios of atmospheric neutrinos in Kamiokande and IMB are due to $\nu_{\mu} \rightarrow \nu_{e}$ oscillations Chooz and Palo Verde should see a clear effect within one year of data taking. However they are not sensitive to $\nu_{\mu} \rightarrow \nu_{\tau}$ oscillations which would still need a confirmation from accelerator experiments.

\subsection{Accelerator neutrinos}

Neutrinos produced at accelerators have some interesting properties for oscillation searches

- neutrino energies above the charged-current threshold for $\nu_{\tau}$ or $\nu_{\mu}$ are available

- the flavour composition of the beam can be varied

- in principle all mixing modes can be tested in appearance mode 
- directed beams can be produced

- neutrino pulses with a well-defined time structure can be produced

In the following, two different energy regimes, resulting in different oscillation searches will be discussed.

\subsubsection{Neutrinos from $\pi$ and $\mu$ decay at rest}

Sending pulses of protons with momentum $800 \mathrm{MeV}$ onto a massive target will mainly produce pions (apart from nuclear break up). These pions are stopped inside the target. The $\pi^{-}$are captured by nuclei. Only a fraction of $10^{-4}$ of the produced $\pi^{-}$decay before they are captured. In contrast, the $\pi^{+}$decay at rest. The muons which are produced come to rest and decay on a different time scale defined by their lifetime.

$$
\begin{array}{ll}
\pi^{+} \rightarrow \mu^{+}+\nu_{\mu} & 26 \mathrm{~ns} \\
\mu^{+} \rightarrow e^{+}+\nu_{e}+\bar{\nu}_{\mu} & 2.2 \mu \mathrm{s}
\end{array}
$$

Using this production mechanism one gets a neutrino flux pattern which is completely defined by the two- and three-body decay kinematics of the pion and muon decay as it is shown in figure 8. An oscillation search can be performed in appearance mode for the

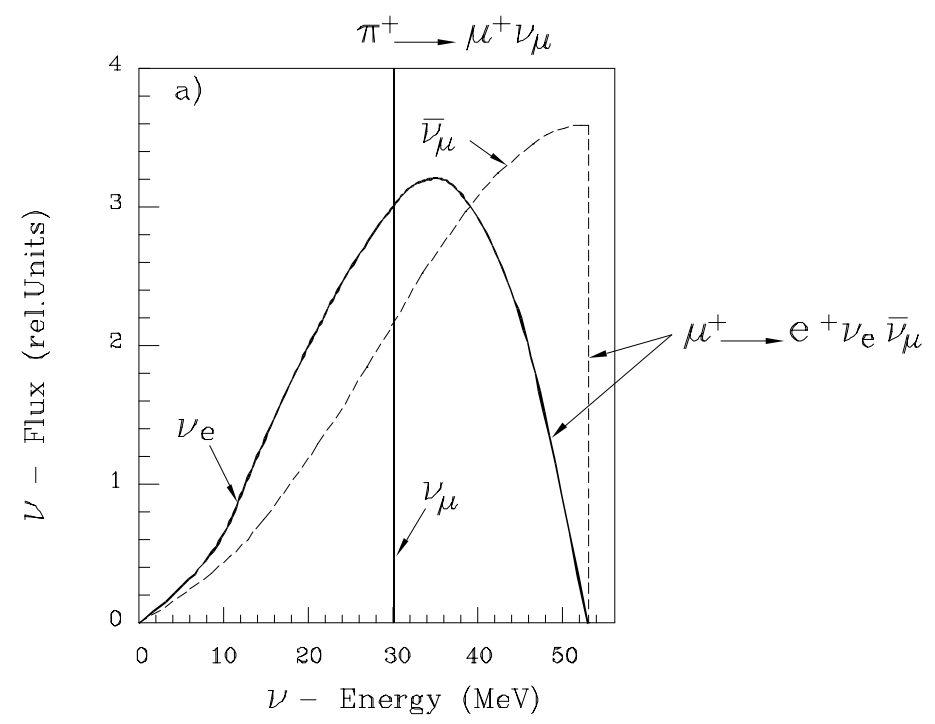

Figure 8: Neutrino flux pattern from pion decay at rest

channels $\bar{\nu}_{\mu} \rightarrow \bar{\nu}_{e}$ because no $\bar{\nu}_{e}$ are produced in the above described scheme. The only source of $\bar{\nu}_{e}$ is $\pi^{-}$decay in flight (followed by a $\mu^{-}$decay) which is suppressed by several orders of magnitude.

The detection principle is quite similar to the detection of reactor $\bar{\nu}_{e}$. One uses the inverse beta decay as given in Eq. 60. The positron is measured, followed by a space and time correlated neutron capture reaction. The photons released in the neutron capture process are measured. Different materials can be used for the neutron capture. The main difference with respect to reactor neutrino detection is the higher energy of the neutrinos from muon decay. The signal is a positron of about 30 to $50 \mathrm{MeV}$ energy which corresponds to the main part of the $\bar{\nu}_{\mu}$ spectrum which would signal $\bar{\nu}_{\mu}-\bar{\nu}_{e}$ oscillations (see figure 8 ). 


\begin{tabular}{||c|c|c||}
\hline & KARMEN & LSND \\
\hline data taking time & $1990-1995$ & $1993-1995$ \\
proton beam momentum & $800 \mathrm{MeV}$ & $800 \mathrm{MeV}$ \\
time structure of beam & $100 \mathrm{~ns}$ pulses & not used \\
integrated beam current & $9122 \mathrm{C}$ & $14772 \mathrm{C}$ \\
target mass & $56 \mathrm{t}$ & $167 \mathrm{t}$ \\
beam dump - detector & $17.5 \mathrm{~m}$ & $30 \mathrm{~m}$ \\
angle proton - neutrino & $85^{\circ}$ & $10^{\circ}$ \\
detection principle & scintillation & scint. and Čerenkov \\
neutron capture & Gadolinium $+\mathrm{p}$ & protons \\
\hline
\end{tabular}

Table 3: Comparison of parameters of KARMEN and LSND [53, 54]

\section{Present status}

Two experiments, KARMEN and LSND, are using currently this technique to search for neutrino oscillations $[53,54]$. A summary of fundamental properties of both experiments is given in table 3 .

KARMEN [53] finds $14.7 \pm 1.2$ events per $\mu \mathrm{s}$ in a window of 1 to $11 \mu \mathrm{s}$ after the proton pulse. The required positron energy is 10 to $50 \mathrm{MeV}$ and a space correlated neutron capture signal has to follow within $500 \mu \mathrm{s}$. The expected background rate is instead $11.0 \pm 0.2$ events per $\mu s$. Therefore an excess of $2.3 \sigma$ has been observed. However neither the energy shape of the excess events nor their time structure follow the expectations of an oscillation signal so the observed rate can be used to deduce the following limit [53]:

$$
P\left(\bar{\nu}_{\mu} \rightarrow \bar{\nu}_{e}\right)<3.75 \cdot 10^{-3} \quad(90 \% \text { C.L. })
$$

Only the last 4 years of data taking have been used to extract this result corresponding to an integrated flux of 7449 Coulombs. One year earlier KARMEN published an oscillation limit [55] which was based on an even smaller number of integrated protons (6021 Coulombs). Somewhat stricter cuts were applied (e.g. a positron energy of at least $30 \mathrm{MeV}$ was required) which led to a zero background measurement and the following limit:

$$
P\left(\bar{\nu}_{\mu} \rightarrow \bar{\nu}_{e}\right)<3.1 \cdot 10^{-3} \quad(90 \% \text { C.L. })
$$

LSND used similar criteria to define its data sample [54]. Only the relative timing with respect to the proton pulse could not be applied. The minimal required positron energy is here $36 \mathrm{MeV}$. Conditions on space and time correlation of the two signals are adapted to the different detector layout and detection principle. Based on the accumulated proton flux quoted in table 3 a signal of 22 events was observed compared to an estimated background of $4.6 \pm 0.6$ events. The positron energy distribution of these events (signal and background) is given in figure 9 in comparison to an oscillation hypothesis. Accepting positron energies between 20 and $60 \mathrm{MeV}$ the total excess over background increases to $52 \pm 18 \pm 8$ events. If this excess is attributed to neutrino oscillations, one obtains [54]:

$$
P\left(\bar{\nu}_{\mu} \rightarrow \bar{\nu}_{e}\right)=(3.1 \pm 1.1 \pm 0.5) \cdot 10^{-3} \quad(90 \% \text { C.L. })
$$

The results is illustrated in figure 10 in comparison with many other measurements in this oscillation channel.

The following differences between the two experiments should be noted: 


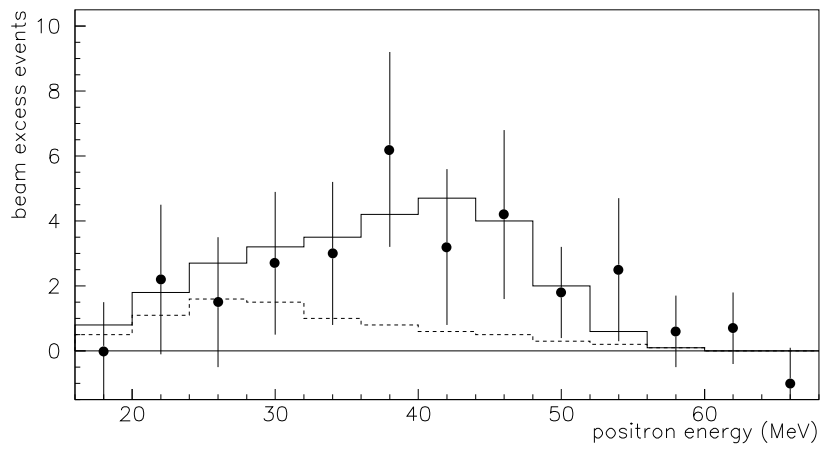

Figure 9: The energy distribution of the LSND beam-off subtracted $\bar{\nu}_{e}$ signal events (data points) compared with the estimated neutrino background (dashed) and the expected distribution for neutrino oscillations at large $\Delta m^{2}$ plus estimated neutrino background (solid) [54]

- The neutrino angle with respect to the proton beam angle is close to zero for LSND. This allows LSND to study as well neutrinos in a higher energy domain coming from pion decay in flight. This has been done [56] but the results disagree with various cross section calculations. The decay in flight is one of the major beam-related background sources for the oscillation search. For KARMEN, with a target almost 90 degrees with respect to the proton beamline, it is impossible to study processes as described in [56], but the background from decay in flight is negligible for the oscillation search.

- Gadolinium, as it is used in KARMEN, is highly efficient for neutron capture. The photons released at the capture reaction have an integrated energy of about $6 \mathrm{MeV}$. For the proton capture reaction, as in LSND, the total photon energy is only $2 \mathrm{MeV}$ which makes it more difficult to distinguish the signal from background.

The pulsed proton beam of the neutron spallation facility ISIS allows KARMEN to distinguish neutrinos of the "fast" pion decay from the ones of the "slow" muon decay. Indeed KARMEN published results on the oscillation channel $\nu_{\mu} \rightarrow \nu_{e}$ whereas LSND did not [53]. The detection of electron neutrinos in KARMEN is done in the following way:

$$
\begin{array}{cll}
\nu_{e}+{ }^{12} C & \rightarrow e^{-}+{ }^{12} N_{\text {g.s. }} & E>17.3 \mathrm{MeV} \\
{ }^{12} N_{\text {g.s. }} & \rightarrow{ }^{12} C+e^{+}+\nu_{e} & \tau=15.9 \mathrm{~ms}
\end{array}
$$

where ${ }^{12} N_{\text {g.s. }}$ denotes the ground state of nitrogen-12. The electron neutrinos are supposed to come from the pion decay at rest. Therefore they are monoenergetic with $E_{\nu}=29.8 \mathrm{MeV}$. So one expects monoenergetic electrons at $12.5=29.8-17.3 \mathrm{MeV}$. The positron from the subsequent beta decay of the nitrogen isotope has a corresponding energy of less than $17.3 \mathrm{MeV}$. A signal of 3 events was observed for a total background estimate of $2.2 \pm 0.3$ events. This can be transformed into a limit [53]:

$$
P\left(\nu_{\mu} \rightarrow \nu_{e}\right)<2.6 \cdot 10^{-2} \quad(90 \% \text { C.L. })
$$


Also for this channel the previously published result was slightly better [55]:

$$
P\left(\nu_{\mu} \rightarrow \nu_{e}\right)<2.4 \cdot 10^{-2} \quad(90 \% \text { C.L. })
$$

Due to the presence of $\nu_{e}$ in the muon decay time window it is possible to perform an independent check of the detection efficiency for them. This has been done and the Michel electron energy shape as well as the total $\nu_{e}$ cross section for the reaction given in Eq. (65) could be reproduced well.

\section{Future projects}

The results from KARMEN and LSND call for further cross checks. The following projects are planned:

- KARMEN performed a major detector upgrade in 1996 to reduce the neutron background, originating from interactions of atmospheric muons [53], which is the limiting factor for the antineutrino channel. To achieve this, an additional active veto layer has been built. Three more years of data taking (1997-1999) should allow reaching the same sensitivity as LSND, as indicated in figure 14 .

- A new accelerator, dedicated to neutron physics has been proposed: the "European Spallation Source" (ESS) which would supply a 60 times higher flux than the existing ISIS facility [53] which is currently used by KARMEN. It should have an equivalent time structure of the beam as ISIS. With a $1000 \mathrm{t}$ water Cerenkov detector one could reach a sensitivity down to $10^{-4}$ in terms of oscillation probability within a few years of running.

- A proposal has been made to use high-energy neutrinos to probe the LSND signal. This will be described in section 4.6.2.

- Some running high energy neutrino experiments (NOMAD, CCFR) are able to probe the LSND signal in the large $\Delta m^{2}$ region. Results will be presented in the following section.

\subsubsection{High-energy neutrinos}

This section is dedicated to neutrinos produced at Multi-GeV proton accelerators which will be called "high-energy neutrinos". They are well above the charged-current threshold for muon-neutrino interactions and for some experiments they are also above the chargedcurrent tau neutrino threshold. Therefore a large variety of oscillation channels can be studied.

\section{Neutrino Beam}

The general scheme of producing high-energy neutrinos is the following: Multi-GeV protons are stopped in a massive target. A hadronic shower develops as result of the protonnucleus interaction. In contrast to the requirement of the beam stop discussed in section 4.6.1, the target dimension must allow the shower to leave the target. A major part of the shower leakage consists of long-lived mesons such as kaons and pions. Mesons of the desired charged are focused by a possibly multi-stage magnetic focusing system. The produced meson beam is sent into a decay tunnel of appropriate length tuned to the mean 
energy of the mesons. A large fraction of the mesons decay in the tunnel dominantly via the following channels :

$$
\begin{array}{lll}
\pi^{+} \rightarrow \mu^{+}+\nu_{\mu} & B r=100 \% \\
K^{+} \rightarrow \mu^{+}+\nu_{\mu} & B r=63.5 \% \\
K^{+} \rightarrow \pi^{o}+\mu^{+}+\nu_{\mu} & B r=3.2 \% \\
K^{+} \rightarrow \pi^{o}+e^{+}+\nu_{e} & B r=4.8 \% \\
K_{L}^{o} \rightarrow \pi^{\mp}+\mu^{ \pm}+\nu_{\mu}\left(\bar{\nu}_{\mu}\right) & B r=27.0 \% \\
K_{L}^{o} \rightarrow \pi^{\mp}+e^{ \pm}+\nu_{e}\left(\bar{\nu}_{e}\right) & B r=38.7 \%
\end{array}
$$

A shielding at the end of the decay tunnel stops the remaining mesons and most of the muons which are produced together with the neutrinos. Sometimes a magnetic field is used in addition to bend the remaining charged particles out of the neutrino beam line. Detectors which are positioned behind the shielding see only the neutrinos from the various decays. The beam composition varies according to the initial proton energy and the specific setup of the beam stop and the focusing section. It is difficult to reduce the fraction of wrong-charge and neutral mesons below the few percent level. Therefore all four flavours $\left(\nu_{e}, \bar{\nu}_{e}, \nu_{\mu}, \bar{\nu}_{\mu}\right)$ will be normally present in the beam. However, the fraction of $\nu_{\tau}$ will be smaller by several orders of magnitude [57] because:

- The $\nu_{\tau}$ can only be produced by heavy mesons, containing the charm or bottom quark; but the production cross section for these particles is much smaller than for kaons and pions.

- The heavy mesons which could produce $\nu_{\tau}$ are too short-lived to reach the beam focusing. Therefore the $\nu_{\tau}$ component of the beam has typically a much larger divergence, which further reduces the flux at the detector location.

- The branching ratio of the decay of these particles into $\nu_{\tau} \mathrm{X}$ is small.

As an example the neutrino beam line at the CERN SPS will be described in more detail [58]. The CERN SPS accelerator supplies $450 \mathrm{GeV}$ protons with a cycle of $14.4 \mathrm{~s}$. Per double extraction, $2 \times 10^{13}$ protons are directed on the target. The detectors are located about 900 meters downstream of the proton target. The mean flight length for neutrinos is about 600 meters taken from the middle of the decay pipe. The relative abundance of the neutrino flavours in the beam and the mean neutrino energies are summarised in table 4 .

\begin{tabular}{||c|c|c||}
\hline & mean Energy & relative rate \\
\hline$\nu_{\mu}$ & $26.9 \mathrm{GeV}$ & $100 \%$ \\
$\bar{\nu}_{\mu}$ & $21.7 \mathrm{GeV}$ & $5.6 \%$ \\
$\nu_{e}$ & $47.9 \mathrm{GeV}$ & $0.7 \%$ \\
$\bar{\nu}_{e}$ & $35.3 \mathrm{GeV}$ & $0.2 \%$ \\
$\nu_{\tau}\left(\bar{\nu}_{\tau}\right)$ & $43.0 \mathrm{GeV}$ & $3 \cdot 10^{-6}$ \\
\hline
\end{tabular}

Table 4: Mean energy and composition of the neutrino beam at the CERN-SPS wideband $\nu$ facility 


\section{Present status}

Due to the beam composition and the high beam energies a variety of possibilities opens to search for oscillations. The most important techniques are summarised below:

$\nu_{\mu} \rightarrow \nu_{e}$ appearance Detection of electron neutrino interactions by using, e.g., the "shower shape" of the event. The sensitivity for $\nu_{\mu} \rightarrow \nu_{e}$ oscillations is limited by the knowledge of the $\nu_{e}\left(\bar{\nu}_{e}\right)$ contamination in the beam and misidentifying other electromagnetic showers (e.g. from $\pi^{o}$ ) as $\nu_{e}$ signatures.

$\nu_{x} \rightarrow \nu_{\tau}$ appearance Detection of the charged-current reaction $\nu_{\tau} N \rightarrow \tau^{-} X$ followed by a $\tau^{-}$decay after $3 \cdot 10^{-13} \mathrm{~s}$. Different concepts have been developed to detect this reaction. The most direct way is the observation of the tau decay $[58,59]$ which requires a high-resolution tracking detector. Another possibility is the analysis of the transverse momentum balance [61], which should be distorted for the $\tau$ due to the escaping neutrinos from the $\tau$ decay. This method of using kinematical information to select $\nu_{\tau}$ events can be combined with a selection of an exclusive channel as it was done in CHARM-II [60]. Due to the very low content of $\nu_{\tau}$ in the beam it is possible to get stringent limits for this type of experiment. The observed limit can be applied independently for the oscillation channel $\nu_{\mu} \rightarrow \nu_{\tau}$ and $\nu_{e} \rightarrow \nu_{\tau}$. The sensitivity for the latter channel is much worse due to the lower $\nu_{e}$ flux.

$\nu_{\mu} \rightarrow \nu_{x}$ disappearance Comparison of $\nu_{\mu}$ charged-current interactions at two distances from the neutrino source. The $\nu_{\mu}$ flux difference between the two locations can be derived. This allows testing the oscillation hypothesis in the channels $\nu_{\mu} \rightarrow \nu_{e}$ and $\nu_{\mu} \rightarrow \nu_{\tau}$.

$\nu_{\mu} \rightarrow \nu_{x}$ mixed mode The ratio of "events with a muon" over "all events" or "events without a muon" is measured. Neutral-current interactions, $\nu_{e}$ charged-current interactions and $\nu_{\tau}$ charged-current interactions with a subsequent non-muonic decay contribute to the class "events without a muon". $\nu_{\mu}$ charged-current events and $\nu_{\tau}$ charged-current events with subsequent muonic decay contribute to the class "events with muon". Various oscillation hypotheses, involving $\nu_{\mu}$, can be tested with different sensitivity.

Table 5 summarises the results of oscillation searches performed at high-energy accelerators. The data of table 5 are illustrated in figure 10. To keep the figure readable only experiments are included which contribute at least at one point of the parameter space to the best possible exclusion limit or which claim evidence for neutrino oscillations. Results from atmospheric, reactor and pion decay-at-rest neutrino experiments have been included as well to make the picture as global as possible. Only the solar neutrino experiments have been left out because they cover an almost entirely different parameter space as illustrated in figure 7. Disappearance experiments, such as reactor experiments, are considered in both possible oscillation channels.

Two experiments are currently taking data on the CERN SPS neutrino beam to search for $\nu_{\tau}$ interactions in the $\nu_{\mu}$ beam : CHORUS [58] and NOMAD [61]. These will be described in more detail.

\section{CHORUS and NOMAD}

As discussed above, high-energy neutrino beams open the unique possibility to search for $\nu_{\mu} \rightarrow \nu_{\tau}$ oscillations in appearance mode due to the low beam contamination by $\nu_{\tau}$ but 


\begin{tabular}{||c|c|c|c|c|c|c|c|c||}
\hline & mean & & \multicolumn{3}{|c|}{$\sin ^{2} 2 \theta$} & \multicolumn{3}{|c||}{$\Delta m^{2}\left(\mathrm{eV}^{2}\right)$} \\
Experiment & Energy & method & \multicolumn{3}{|c|}{$\Delta m^{2} \rightarrow \infty$} & \multicolumn{3}{|c||}{$\sin ^{2} 2 \theta=1$} \\
\cline { 4 - 9 } & $(\mathrm{GeV})$ & & $e \mu$ & $\mu \tau$ & $e \tau$ & $e \mu$ & $\mu \tau$ & $e \tau$ \\
\hline CCFR [62] & 140 & $\nu_{\mu} \rightarrow \nu_{e}$ & 0.0018 & & & 1.6 & & \\
SKAT [63] & 7 & $\nu_{\mu} \rightarrow \nu_{e}$ & 0.0025 & & & 10. & & \\
BNL-E776 [64] & 5 & $\nu_{\mu} \rightarrow \nu_{e}$ & 0.003 & & & 0.075 & & \\
BNL-E734 [65] & 5 & $\nu_{\mu} \rightarrow \nu_{e}$ & 0.0034 & & & 0.43 & & \\
CHARM II [66] & 25 & $\nu_{\mu} \rightarrow \nu_{e}$ & 0.0056 & & & 0.85 & & \\
CHARM [67] & 1.5 & $\nu_{\mu} \rightarrow \nu_{e}$ & 0.008 & & & 0.19 & & \\
BEBC [68] & 1.5 & $\nu_{\mu} \rightarrow \nu_{e}$ & $0.013^{3}$ & & & 0.09 & & \\
\hline FNAL-E531 [59] & 25 & $\nu_{x} \rightarrow \nu_{\tau}$ & & 0.005 & 0.18 & & 0.9 & 9.0 \\
CHARM II [60] & 25 & $\nu_{x} \rightarrow \nu_{\tau}$ & & 0.008 & 0.4 & & 1.5 & 10. \\
BEBC [69] & 25 & $\nu_{e} \rightarrow \nu_{\tau}$ & & & 0.36 & & & 44. \\
\hline Loverre [70] & 50 & $\nu_{\mu} \rightarrow \nu_{x}$ & 0.003 & 0.006 & & 2.4 & 3.3 & \\
CCFR [71] & 140 & $\nu_{\mu} \rightarrow \nu_{x}$ & 0.0038 & 0.0081 & & 1.6 & 1.4 & \\
CDHS [72] & 1.5 & $\nu_{\mu} \rightarrow \nu_{x}$ & $0.053^{2}$ & $0.053^{2}$ & & 0.25 & 0.25 & \\
CHARM [67] & 1.5 & $\nu_{\mu} \rightarrow \nu_{x}$ & $0.17^{1}$ & $0.17^{1}$ & & 0.28 & 0.28 & \\
BEBC [73] & 25 & $\nu_{e} \rightarrow \nu_{x}$ & 0.01 & & 0.07 & 1.7 & & 10. \\
\hline
\end{tabular}

Table 5: Summary of negative searches for neutrino oscillations from various accelerator experiments (1: at $\Delta m^{2}=0.29 \mathrm{eV}^{2} ; 2$ : at $\Delta m^{2}=2.5 \mathrm{eV}^{2} ; 3:$ at $\Delta m^{2}=2.2 \mathrm{eV}^{2}$ )

sufficiently high-energy neutrinos to operate above the kinematical threshold for chargedcurrent $\nu_{\tau}$ interactions $\left(m_{t h r}=m_{\tau}+m_{\tau}^{2} / 2 m_{N}=3.5 \mathrm{GeV}\right)$. The accessible parameter range is

$$
\begin{array}{ll}
\sin ^{2} 2 \theta & >10^{-4} \\
\Delta m^{2} & >1 \mathrm{eV}^{2} .
\end{array}
$$

A motivation for an oscillation search in this parameter range can be obtained with the straightforward assumptions that:

- neutrino masses follow a hierarchy comparable to the charged leptons. This means the tau neutrino would be the heaviest neutrino.

- neutrinos account for the missing hot dark matter in the universe with a mass of 1 to $10 \mathrm{eV}$.

NOMAD An elevation view of the NOMAD detector is given in figure 11. The target volume consists of 45 drift chambers. They are made of a self-supporting structure of polystyrene/polypropylene foam which makes up the target mass of 3 tons. These drift chambers achieve a precision of $\sigma_{y}=200 \mu \mathrm{m}$ and $\sigma_{x}=2 \mathrm{~mm}$. Each chamber is equivalent to 0.02 radiation length which resolves the photon conversion point from the interaction vertex. This is important to distinguish electrons from photon conversion and electrons from the neutrino vertex. Data taking with the completely assembled detector started in August 1995. The drift chambers are followed by 5 modules of a transition radiation detector (TRD) to separate electrons from hadrons. After the TRD there is a preshower detector followed by a calorimeter for energy measurement of electromagnetic showers. All 


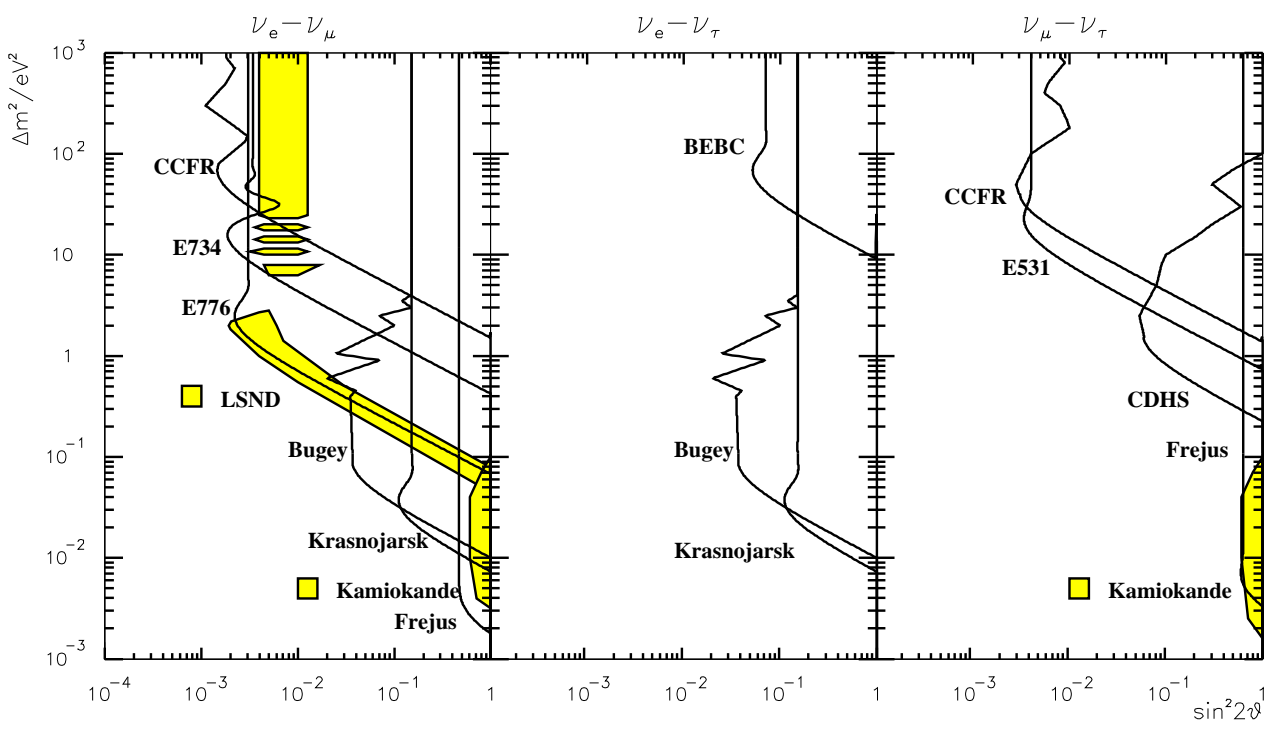

Figure 10: Comparison of results from experiments at accelerators, reactors and from atmospheric neutrino studies for all three oscillation channels. The shaded areas are positive results as reported from LSND and Kamiokande. No distinction has been made between $\nu$ and $\bar{\nu}$

the above mentioned detector parts are located inside a dipole magnet which supplies a field of 0.4 Tesla perpendicular to the beam axis. Therefore the momentum determination of charged tracks from the primary vertex using their curvature in the magnetic field should be possible with high precision. The NOMAD detector is completed by trigger planes, muon chambers and front and back calorimeters which are the only components outside the magnet coils.

In 1995 and 1996 about 500,000 charged-current $\nu_{\mu}$ interactions have been recorded on tape. So far $20 \%$ of the data have been analysed to study the event kinematics, which is the key point of the NOMAD oscillation search. The $\tau$-decay searches in several exclusive channels are under development:

$$
\begin{array}{ll}
\tau^{-} \rightarrow e^{-} \bar{\nu}_{e} \nu_{\tau} & 17.8 \% \\
\tau^{-} \rightarrow \mu^{-} \bar{\nu}_{\mu} \nu_{\tau} & 17.4 \% \\
\tau^{-} \rightarrow \pi^{-} \nu_{\tau} & 11.3 \% \\
\tau^{-} \rightarrow \pi^{-} \pi^{o} \nu_{\tau} & 25.2 \%
\end{array}
$$

Preliminary results have been presented [74]. For large $\Delta m^{2}$

$$
\sin ^{2} 2 \theta>4 \cdot 10^{-3}
$$

can be excluded. Based on the powerful electron identification, limits for the channel $\nu_{\mu}-\nu_{e}$ have been derived as well.

$$
\sin ^{2} 2 \theta>2 \cdot 10^{-3} \text { for } \Delta m^{2} \rightarrow \infty
$$




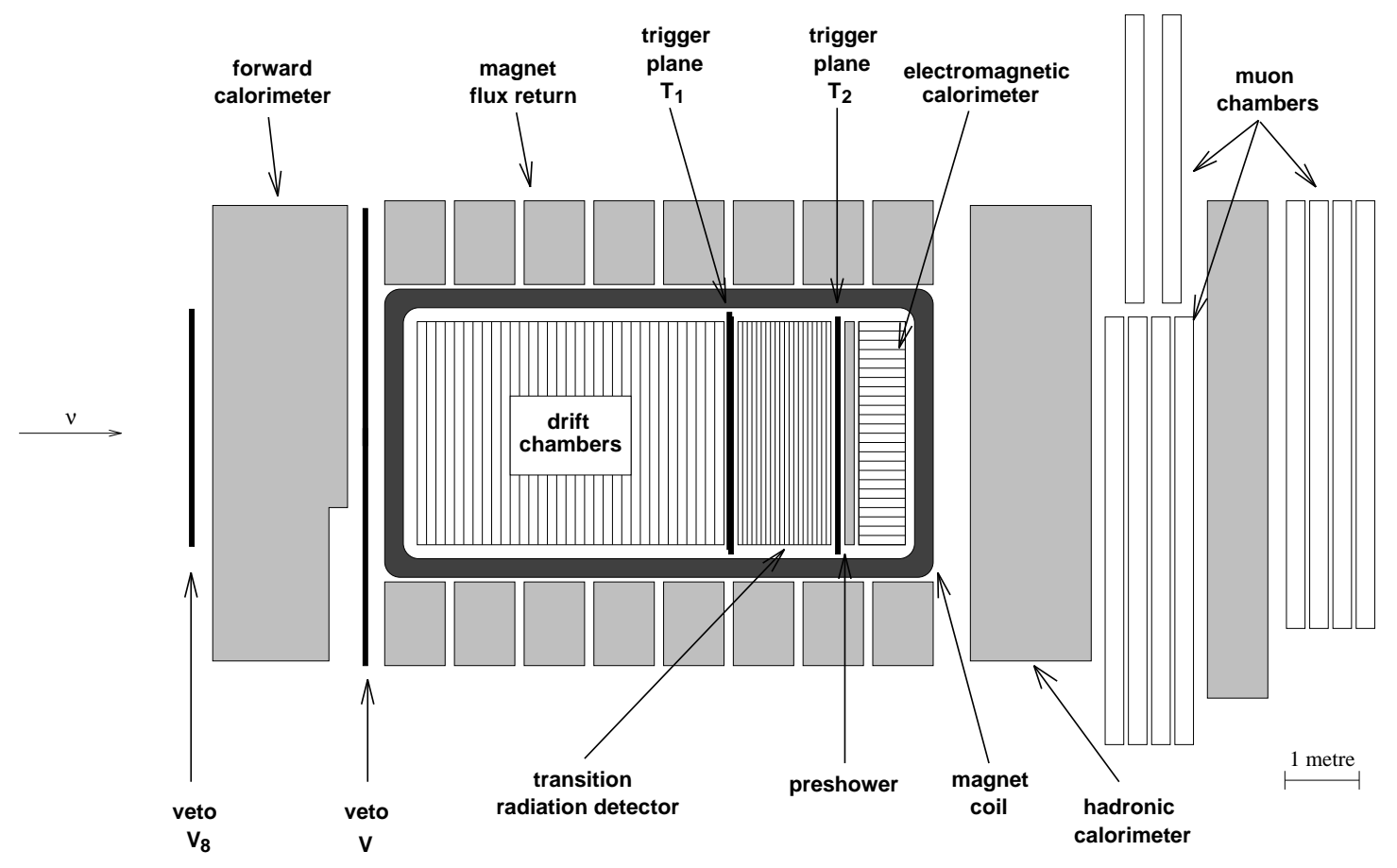

Figure 11: Elevation view of the NOMAD detector

At least one further year of data taking is foreseen. The sensitivity for the $\nu_{\tau}$ appearance channels after the full analysis is indicated in figure 14 .

CHORUS The concept of the CHORUS experiment is based on the detection of the $\tau$ decay vertex. This requires a high tracking resolution due to the short life time of the $\tau$ lepton. Nuclear emulsion supplies a three-dimensional resolution of $1 \mu \mathrm{m}$ and it integrates the properties of an active detector and a heavy target which is best suited for neutrino experiments. The disadvantage of the long procedure of the emulsion analysis after exposure has been overcome by using fully automatic scanning. In the CHORUS experiment an emulsion target of $800 \mathrm{~kg}$ is used. Scintillating fibre trackers give precise tracking close to the emulsion target and define the entry point of the tracks in the emulsion for automatic scanning with a resolution of $\Delta x=200 \mu \mathrm{m}$ and $\Delta \theta=3 \mathrm{mrad}$. Figure 12 gives a schematic view of this procedure. A hexagonal air-core magnet determines the momenta of hadronic tracks. All above mentioned parts of the detector are contained in a cool-box $\left(5^{\circ} \mathrm{C}\right)$ which supplies a stable environment to slow down fading of the emulsion and aging of the fibres. The tracker region is followed by a high-resolution calorimeter, built from scinillation fibers embedded in lead, for energy measurement of hadronic and electromagnetic showers. It provides an energy resolution of $13 \% / \sqrt{E}$ for electrons and $30 \% / \sqrt{E}$ for hadrons. A muon spectrometer determines charge and momenta of muons. The detector is completed by several veto and trigger hodoscope planes. An elevation view of the whole detector is shown in figure 13.

CHORUS started data taking in 1994. After 2 years of exposure 320,000 chargedcurrent $\nu_{\mu}$ interactions had been accumulated in the emulsion target which was replaced by a fresh target of the same weight. In 1996 and 1997 a second 2 year exposure is foreseen. Meanwhile the emulsion target of the first two years of data taking is being analysed. The current analysis concentrates on data taken in 1994 for the muonic decay channel of the 


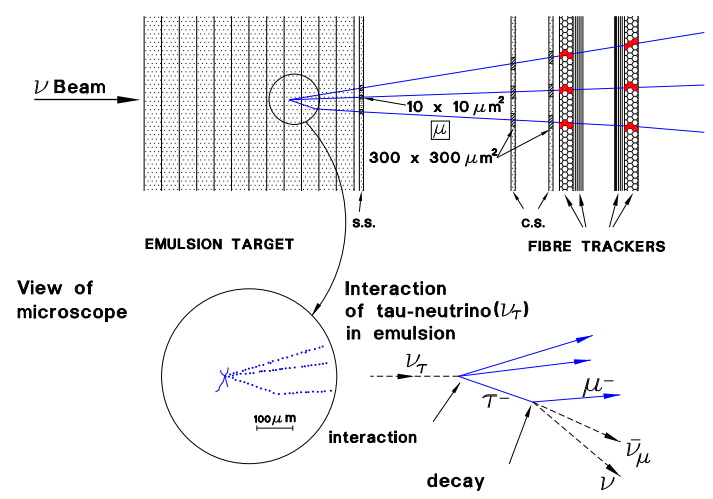

Figure 12: Concept for the detection of the reaction $\nu_{\tau} N \rightarrow \tau^{-} X$ in CHORUS

$\tau:$

$$
\tau^{-} \rightarrow \mu^{-} \bar{\nu}_{\mu} \nu_{\tau} \quad 17.4 \%
$$

So far, 50,000 charged-current events have been analysed, leading to about 10,000 located interaction vertices in the emulsion. The muon tracks in these events have been carefully analysed. For $\tau$ decays one would expect to find in most cases a significant kink. No events could be found so far [75] allowing an exclusion of $\nu_{\mu}-\nu_{\tau}$ oscillations for

$$
\sin ^{2} 2 \theta>8 \cdot 10^{-3}
$$

in the limit of large $\Delta m^{2}$.

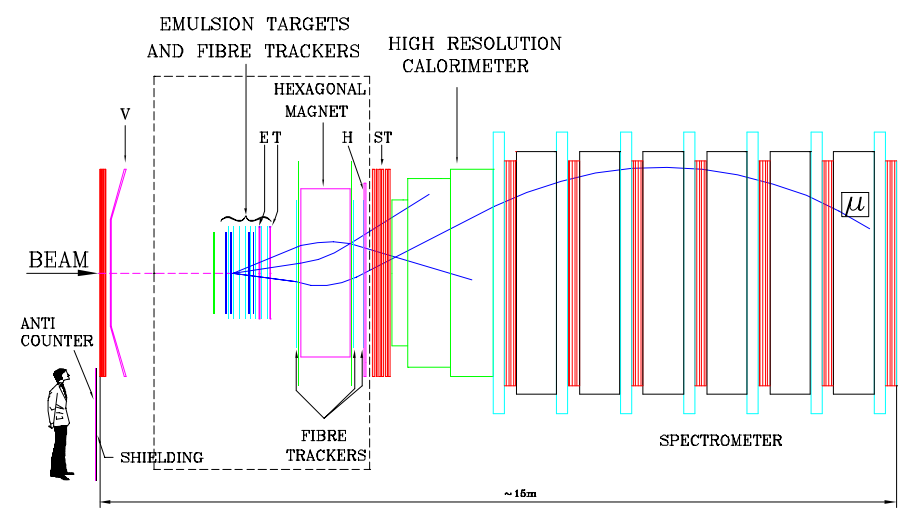

Figure 13: Elevation view of the CHORUS detector

Extrapolating this result to an analysis of the full data sample and including of the hadronic decay channels

$$
\begin{array}{lr}
\tau^{-} \rightarrow h^{-}\left(n \pi^{o}\right) \nu_{\tau} & 49.8 \% \\
\tau^{-} \rightarrow h^{-} h^{-} h^{+}\left(n \pi^{o}\right) \nu_{\tau} & 14.9 \%
\end{array}
$$


leads to a sensitivity of

$$
\begin{array}{ll}
\sin ^{2} 2 \theta \quad 2 \cdot 10^{-4} & \text { for } \\
\Delta m^{2}>0.4 \mathrm{eV}^{2} \rightarrow \infty \\
\text { for } & \sin ^{2} 2 \theta=1
\end{array}
$$

as indicated in figure 14 .

\section{Future projects}

New proposals of accelerator neutrino oscillation experiments take three different approaches:

Test of LSND signal The limiting factor for high-energy accelerator oscillation searches in the domain of KARMEN and LSND is the presence of $\nu_{e}$ and $\bar{\nu}_{e}$ in the beam coming from kaon decays (see Eq. 68). To overcome this problem an anti-tagging mechanism [76] has been suggested. The idea is to detect the electrons, which accompany the production of $\nu_{e}$ and $\bar{\nu}_{e}$ in the decay tunnel by Cerenkov light. This would allow creating a veto signal for the experiment, resulting in a reduced prompt $\nu_{e}, \bar{\nu}_{e}$ flux by several orders of magnitude. So one can reach and improve the sensitivity of the two running experiments remarkably. The proposed experiment would operate at the CERN PS accelerator at about $8 \mathrm{GeV}$ proton energy. The estimated sensitivity for 2 years running is indicated in figure 14 .

Further study of $\nu_{\mu}-\nu_{\tau}$ oscillations Following the motivation for CHORUS and NOMAD some new proposals have been made to improve the sensitivity beyond $\sin ^{2} 2 \theta=$ $10^{-4}$. The COSMOS experiment at Fermilab [77] proposes a setup similar to CHORUS. It is planned to run at the new Main Injector for the Tevatron supplying $120 \mathrm{GeV}$ protons which is currently under construction. The experiment could start around the year 2000 . The sensitivity for 2 years data taking is estimated to be:

$$
\begin{array}{ll}
\sin ^{2} 2 \theta & >2.8 \cdot 10^{-5} ; \quad \Delta m^{2} \rightarrow \infty \\
\Delta m^{2} & >0.13 \mathrm{eV}^{2} ; \quad \sin ^{2} 2 \theta=1 .
\end{array}
$$

NAUSICAA [78] and TENOR [79] try to integrate the concepts of CHORUS and NOMAD. Both propose to use a large dipole magnet to obtain precise momentum information for a large fraction of the charged particles from the neutrino interaction. The neutrino beam at the CERN SPS should be used with slightly reduced proton energy (e.g. $350 \mathrm{GeV}$ ) to reduce further the background from $\nu_{\tau}$ in the beam, which becomes important at the sensitivity which is aimed for by these experiments. Data taking could start around 2000 . NAUSICAA is a combination of silicon strip detectors and passive target material. The $\tau$ lepton decay point could be identified by indirect methods such as impact-parameter measurement. The estimated sensitivity for 4 years running is comparable to COSMOS (for 2 years running). TENOR explores further the emulsion technique. A 5 ton emulsion target is proposed. A new feature is the use of interface emulsion sheets in the tracking region to improve the momentum resolution of charged tracks. The estimated sensitivity for two years running is another factor three better than COSMOS. As an example of these three proposals the COSMOS values are indicated in figure 14. 
Test of the atmospheric neutrino anomaly As it was discussed in section 4.4 this anomaly allows 2 neutrino oscillation hypotheses as possible explanation : $\nu_{\mu}-\nu_{e}$ or $\nu_{\mu}-\nu_{\tau}$. Whereas the first possibility will be checked by the new reactor experiments Chooz [50] and Palo Verde [51] within two years, the second possibility can only be tested with long baseline accelerator experiments. Table 6 summarises proposed and scheduled projects.

\begin{tabular}{||c|c|c|c|c||}
\hline$\nu$ source & $\begin{array}{c}\text { proton } \\
\text { energy }\end{array}$ & detector & distance & weight \\
\hline KEK & $\begin{array}{c}12 \mathrm{GeV} \\
50 \mathrm{GeV}\end{array}$ & $\begin{array}{c}\text { SuperK } \\
\text { SuperK }\end{array}$ & $250 \mathrm{~km}$ & $50 \mathrm{~km}$ \\
& $50 \mathrm{kt}$ \\
\hline Fermilab & $120 \mathrm{GeV}$ & MINOS & $735 \mathrm{~km}$ & $10 \mathrm{kt}$ \\
\hline \multirow{3}{*}{ CERN } & $450 \mathrm{GeV}$ & ICARUS & $735 \mathrm{~km}$ & $5 \mathrm{kt}$ \\
& $450 \mathrm{GeV}$ & NOE & $735 \mathrm{~km}$ & $4 \mathrm{kt}$ \\
& $450 \mathrm{GeV}$ & RICH & $735 \mathrm{~km}$ & $27 \mathrm{kt}$ \\
\hline
\end{tabular}

Table 6: Long baseline accelerator projects which are proposed or scheduled

The only fully approved and scheduled project is the $12 \mathrm{GeV}$ KEK beamline. The water Čerenkov detector (Superkamiokande) [27] is operational since April 1996 and the beamline is scheduled for the end of 1998, so the experiment could start in 1999. The differentiation of muon and electron signals will be done in the same way as for atmospheric neutrino interactions. However, $12 \mathrm{GeV}$ protons produce a neutrino beam with an average energy of about $1 \mathrm{GeV}$, which is below the $\tau$ threshold. The oscillation channel $\nu_{\mu}-\nu_{\tau}$ can therefore only be studied in the disappearance mode $\nu_{\mu}-\nu_{x}$ which seriously limits the sensitivity in the mixing angle. To overcome this problem a new proton accelerator of $50 \mathrm{GeV}$ is proposed. It is awaiting approval in 1997 and could be constructed by 2003 .

The MINOS detector [80] will be installed in the SOUDAN mine, $735 \mathrm{~km}$ away from Fermilab. It will be a sandwich structure of magnetised iron toroids and streamer tubes. Among various methods which are discussed in the proposal the best sensitivity for an oscillation signal can be reached studying the "event with a muon" over "event without a muon" rate as discussed in section 4.6.2. The neutrino beamline will be related to the new Main Injector for the Tevatron which is currently under construction. Therefore data taking could start around 2000 pending approval of the project.

ICARUS [81] proposes to use liquid Argon-Methane TPC's allowing a resolution comparable to bubble chambers. A 600 ton prototype has been approved to be installed in the Gran Sasso laboratory.

NOE [82] proposes to use calorimetric modules composed of iron/concrete and scintillating fibres. The detection method for oscillations is similar to MINOS. The detector is, similar to ICARUS, proposed for installation in the Gran Sasso underground laboratory.

RICH [83] is designed as a water Čerenkov detector equipped with a spherical mirror to obtain clean ring signals which would improve the particle identification and which allows to deal with more complicated topologies as "single ring" events compared to conventional water Cerenkov detectors. Due to the size of the detector, installation above ground is foreseen. The dramatic increase of the atmospheric muon rate in such a location could be compensated using the spill structure of the neutrino beam.

The sensitivity for $\Delta m^{2}$ for maximal mixing is close to $10^{-3} \mathrm{eV}^{2}$ for all proposed exper- 
iments for both oscillation channels which are considered $\left(\nu_{\mu}-\nu_{e}\right.$ and $\left.\nu_{\mu}-\nu_{\tau}\right)$. Therefore all of them would be able to probe the parameter region favoured by the Kamiokande result. The sensitivity in $\sin ^{2} 2 \theta$ for large $\Delta m^{2}$ varies considerably according to the different detector masses, running periods etc. It ranges from 0.4 for the $12 \mathrm{GeV}$ KEK project to $4 \cdot 10^{-4}$ for the RICH proposal in the $\nu_{\mu}-\nu_{\tau}$ channel and 0.2 to $10^{-3}$ for the $\nu_{\mu}-\nu_{e}$ channel. As an example of these experiments, the values from the NOE proposal [82] are shown in figure 14.

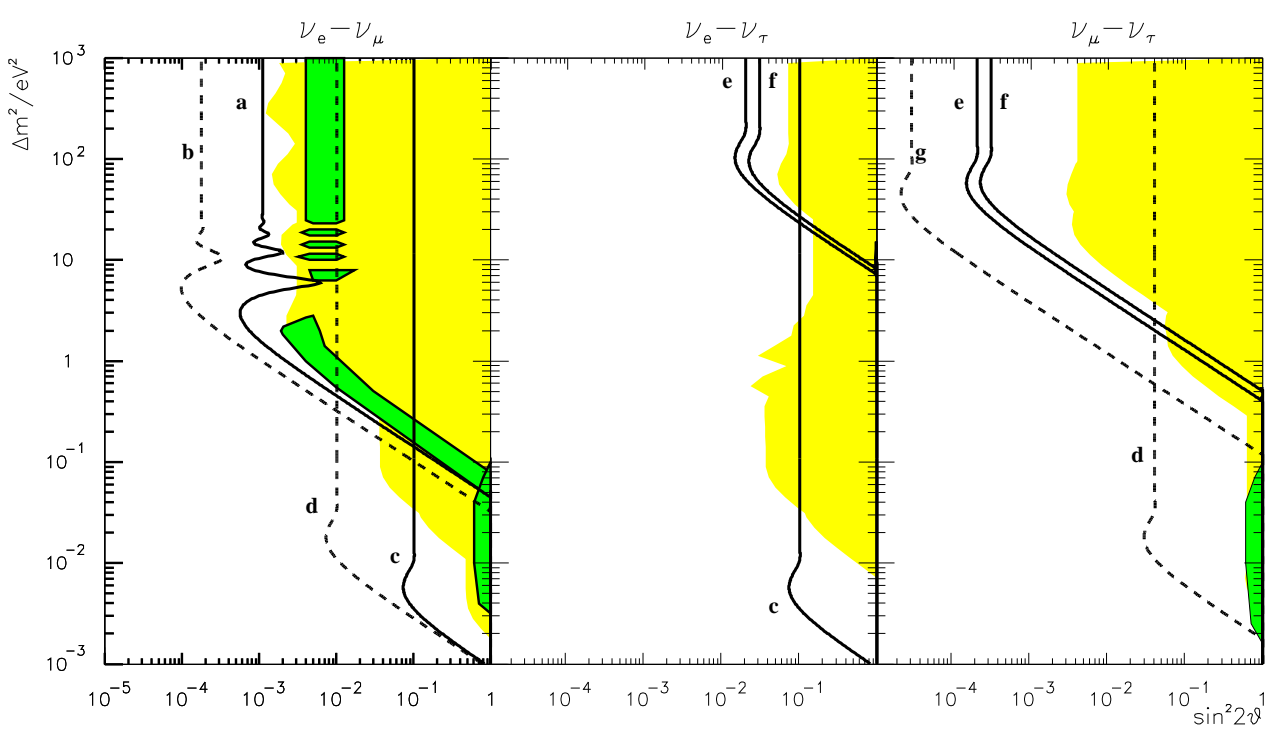

Figure 14: Sensitivity of running or near future experiments. Shaded areas: existing results (see figure 10); full lines: expected final results of running experiments; dashed lines: proposed experiments; a) KARMEN b) anti-tagging c) reactor long baseline (example Chooz) d) accelerator long baseline (example NOE) e) CHORUS f) NOMAD g) future $\nu_{\tau}$ appearance (example COSMOS)

\section{Conclusion}

The present situation is complex. If one takes all experimental results, which show deviations from the expected standard model behaviour as evidence for neutrino oscillations, one would need 3 different mass scales for $\Delta m^{2}$ :

$$
\begin{array}{ll}
\text { LSND : } & \Delta m^{2}>1 \mathrm{eV}^{2} \\
\text { Kamiokande atm. } \nu: & \Delta m^{2} \approx 10^{-2}-10^{-3} \mathrm{eV}^{2} \\
\text { Solar neutrino deficit : } & \text { MSW solution } \Delta m^{2} \approx 10^{-4}-10^{-6} \mathrm{eV}^{2} \\
& \text { vacuum solution } \Delta m^{2} \approx 10^{-10}-10^{-11} \mathrm{eV}^{2}
\end{array}
$$

As shown in Eq. 28 three neutrino flavours produce only two independent mass scales. Therefore it is not possible to find a set of mass and mixing parameters which would agree with all experimental results in this framework. A possible solution is the extension of the theory and the introduction of sterile neutrinos. A more conventional alternative is the 
assumption that one or more of the experimental results under discussion here is caused by effects other than neutrino oscillations. Modifications of the calculated neutrino flux and cross sections could contribute as well as undetected systematic errors of the experiments. The present situation calls for new experiments which would independently check the existing results preferably even with different techniques to exclude the possibility of repeating the same systematic error. As has been shown, such experiments are running or at least scheduled for the near future. Therefore one might hope for new hints to solve the neutrino mass and mixing puzzle within a few years.

\section{Acknowledgements}

I would like to thank Klaus Winter, who gave me the inspiration for this work. I am also grateful to D. Saltzberg, Ch. Weinheimer, F. Cassol and W. Brunner for helpful discussions and careful reading of the manuscript.

\section{References}

[1] LEP Electroweak Working Group, preprint CERN-PPE/95-172 (1995).

[2] A.Klypin et al. preprint ASTRO-PH/9502062 (1995); R.Nolthenius et al., Astrophys. J. 422 (1994) L45; D.Schlegel et al., Astrophys. J. 427 (1994) 512; A.Klypin et al., Astrophys. J. 416 (1993) 1.

[3] G.F.Smoot et al., Astrophys. J. 396 (1992) 3, 155-B5; 396 (1992) L1, 160-B1.

[4] M.L.Fisher et al., Astrophys. J. 388 (1992) 242, 53-C11.

[5] Ch.Weinheimer et al., Phys. Lett. B300 (1993) 210.

[6] A.I.Belesev et al., Phys. Lett. B350 (1995) 263.

[7] V.M.Lobashev, Proc. of Neutrino 96 (Helsinki, 1996) in press.

[8] J.Bonn, Proc. of Neutrino 96 (Helsinki, 1996) in press.

[9] R.M.Barnett et al., Phys. Rev. D54 (1996) 22.

[10] B.Jeckelmann et al., Nucl. Phys. A457 (1986) 709.

[11] B.Jeckelmann et al., Phys. Lett. B335 (1994) 326.

[12] K.Assamagan et al., Phys. Rev. D53 (1996) 6065, K.Assamagan et al., Phys. Lett. B335 (1994) 231.

[13] H.Albrecht et al., ARGUS coll., Phys. Lett. B292 (1992) 221.

[14] A. Gregorio, ALEPH coll., Proc. of Neutrino 96 (Helsinki, 1996) in press.; D. Buskulic et al., ALEPH coll., Phys. Lett. B349 (1995) 585.

[15] G. Alexander et al., OPAL coll., Preprint CERN-PPE-96-042, submitted to Phys. Lett. B.

[16] S.R.Elliot et al., Phys. Rev. Lett. 59 (1987) 2020.

[17] T. Kirsten et al., Proc. of Nuclear Beta Decays and Neutrino (1986) 81.

[18] H. Klapdor-Kleingrothaus, Proc. of Neutrino 96 (Helsinki, 1996) in press.

[19] B.Pontecorvo, Sov. Phys. JETP 6 (1958) 429.

[20] Z.Maki et al., Progr. Theor. Phys. 28 (1962) 870.

[21] L.Wolfenstein, Phys. Rev. D17 (1978) 2369; D20 (1979) 2634. S.P.Mikheyev and A.Yu.Smirnov, Yad. Fiz. 42 (1985) 1441; Nuo. Cim. 9C (1986) 17. 
[22] S.M.Bilenky and S.T.Petcov Rev. Mod. Phys. 59 (1987) 671;

T.K.Kuo and J.Pantaleone, Rev. Mod. Phys. 61 (1989) 937.

[23] J.N. Bahcall, M.H. Pinsonneault, Rev. Mod. Phys. 67 (1995) 781.

[24] T.Kirsten, GALLEX coll., Proc. of Neutrino 96 (Helsinki, 1996) in press.

[25] V.N.Gavrin, SAGE coll., Proc. of Neutrino 96 (Helsinki, 1996) in press.

[26] K.Lande, Homestake coll., Proc. of Neutrino 96 (Helsinki, 1996) in press.

[27] Y.Suzuki, Kamiokande coll., Proc. of Neutrino 96 (Helsinki, 1996) in press.

[28] Y.Suzuki, SuperKamiokande coll., Proc. of ICHEP (Warsaw, 1996) in press.

[29] R.Davis et al., Homestake coll., Proc. of 21st Int. Cosmic ray Conf. (Adelaide,1990) 143.

[30] J.N.Bahcall, Phys. Rev. D44 (1991) 1644.

[31] V.Berezinsky, G.Fiorentini and M.Lissia, Phys. Lett. B365 (1996) 185; K.H.Heeger and R.G.H.Robertson, Phys. Rev. Lett. 77 (1996) 3720.

[32] P.F.Harrison, D.H.Perkins, W.G.Scott, Phys.Lett. B349 137.

[33] N.Hata and P.Langacker, Phys. Rev. D50 (1994) 632; Phys. Rev. D52 (1995) 420.

[34] H.H. Chen, SNO coll., Nucl. Instr. Meth. A264 (1988) 48.

[35] R.B.Vogelaar, Borexino coll., Proc. of Neutrino 96 (Helsinki, 1996) in press.

[36] E.Belotti et al., GNO-proposal (1996) http://kosmopc.mpi-hd.mpg.de/GALLEX/GALLEX.HTML.

[37] T.K.Gaisser and T.Stanev, Phys. Rev. B39 (1989) 3532; M.Treichel Z. Phys. C54 (1992) 469; P.Lipari Astrop. Phys. 1 (1993) 195; M.Kawasaki and S.Mizuta, Phys. Rev. D43 (1991) 2900; D.Perkins Astrop. Phys. 2 (1994) 249.

[38] K.Daum et al., Frejus coll. Z. Phys. C66 (1995) 417; Ch. Berger et al., Phys. Lett. B245 (1990) 305, Phys. Lett. B227 (1989) 489.

[39] M.Aglietta et al., Nusex coll., Europhys. Lett. 8 (1989) 611.

[40] W.W.M.Allison et al., Soudan2 coll., Preprint hep-ex/9611007 (SPIRES-SLAC database), submitted to Phys. Lett.

[41] D.Caspers et al., IMB coll., Phys. Rev. Lett. 66 (1991) 2561.

[42] K.S.Hirata et al., Kamiokande coll., Phys. Lett. B280 (1992) 146.

[43] Y.Fukuda et al., Kamiokande coll., Phys. Lett. B335 (1994) 237.

[44] D.Saltzberg, Phys. Lett. B355 (1995) 499.

[45] F.Reines and C.L.Cowan, Phys. Rev. 92 (1953) 830.

[46] A.A.Kuvshinnikov et al., Rovno coll., Yad. Fiz. 52 (1990) 300.

[47] G.Zacek et al., Gösgen coll., Phys. Rev. D34 (1986) 2621.

[48] G.S.Vidyakin et al., Krasnojarsk coll., JETP Lett. 59 (1994) 364.

[49] B.Achkar et al., Bugey coll., Nucl. Phys. B434 (1995) 503. M.Abbes et al., preprint LAPPEXP-95.07 (1995), submitted to Nucl. Instr. Meth.; Y.Declais et al., preprint LAPP-EXP96.02 (1996), submitted to Phys. Lett. B

[50] C.Bemporad, Chooz coll. Proc. of Neutrino 96 (Helsinki, 1996) in press.

[51] G.Gratta, Palo Verde coll., Proc. of Neutrino 96 (Helsinki, 1996) in press.

[52] Chooz coll., Proc. of Workshop of future of Neutrino physics, (Frascati, 1996) in press.

[53] K.Eitel, KARMEN coll., Proc. of the 8th Rencontres de Blois, (Blois, 1996), in press. 
[54] C.Athanassopoulos et al., LSND coll., preprint LA-UR-96-1582 (1996);

C.Athanassopoulos et al., LSND coll., preprint LA-UR-96-1326 (1996), submitted to Phys. Rev. C.

[55] B.Armbruster et al., KARMEN coll., Nucl. Phys. B38 (1995) 235.

[56] M.Albert et al., LSND coll., Phys. Rev. C51 (1995) 1065.

[57] B.Van de Vyver and P.Zucchelli; preprint CERN-PPE/96-113 (1996), submitted to Nucl. Instr. Meth.

[58] M.de Jong et al., CHORUS coll., preprint CERN-PPE/93-131 (1993).

[59] N.Ushida et al., FNAL-E531 coll., Phys. Rev. Lett. 57 (1986) 2897.

[60] M.Gruwe et al., CHARM II-coll., Phys. Lett. B309 (1993) 463.

[61] P.Astier et al., NOMAD coll., preprint CERN-SPSLC/91-21 (1991), SPSC/P261.

[62] A.Romosan et al., CCFR coll., preprint NEVIS-1529 (1996).

[63] V.V.Ammososv et al., SKAT coll., Z. Phys. C40 (1988)487.

[64] L.Borodovsky et al., BNL-E776 coll., Phys. Rev. Lett. 68 (1992) 274.

[65] L.A.Ahrens et al. BNL-E734 coll. Phys. Rev. D31 (1985) 2732.

[66] P.Vilain et al.,CHARM II coll., Z. Phys. C64 (1994) 539.

[67] F.Bergsma et al.,CHARM coll., Z. Phys. C40 (1988) 171.

[68] C.Angelini et al., BEBC coll., Phys. Lett. B179 (1986) 307.

[69] M.Talebzadeh et al., BEBC coll., Nucl. Phys. B291 (1987) 503.

[70] P.F.Loverre, Phys. Lett. B370 (1996) 156.

[71] K.S.McFarland et al., CCFR coll., Phys. Rev. Lett. 75 (1995) 3993.

[72] F.Dydak et al., CDHS coll., Phys. Lett. B134 (1984) 281.

[73] O.Erriquez et al., BEBC coll., Phys. Lett. B102 (1981) 73.

[74] Thomas Weiße et al., NOMAD coll., Proc. of Rencontres de Physique 97 (La Thuile, 1997) in press.

[75] P.Migliozzi et al., CHORUS coll., Proc. of Rencontres de Physique 97 (La Thuile, 1997) in press.

[76] L.Ludovici and P.Zucchelli; preprint CERN-PPE/96-181 (1996).

[77] K.Kodama et al., COSMOS coll., FERMILAB-PROPOSAL-P-803 (1993).

[78] J.-J.Gomez-Cadenas et al., (NAUSICAA), preprint CERN-PPE/95-177 (1995).

[79] A.Ereditato et al., (TENOR), preprint CERN-PPE/96-106 (1996).

[80] E.Ables et al., MINOS coll., FERMILAB-PROPOSAL-P-875, (1995).

[81] P.Benetti, ICARUS coll., Nucl. Phys. B35 (1994) 280.

[82] M.Ambrosio et al., NOE coll., Nucl. Instr. Meth. A363 (1995) 604.

[83] T.Ypsilantis et al., RICH coll., preprint CERN-LAA/96-01 (1996). 